\title{
Disruption of staphylococcal aggregation protects against lethal lung injury
}

\author{
Jaime L. Hook,, ${ }^{1}$ Mohammad N. Islam,, ${ }^{1}$ Dane Parker, ${ }^{2}$ Alice S. Prince, ${ }^{2}$ Sunita Bhattacharya, ${ }^{1,2}$ and Jahar Bhattacharya ${ }^{1,3}$ \\ 'Lung Biology Laboratory, Division of Pulmonary, Allergy, and Critical Care Medicine, Department of Medicine, 'Department of Pediatrics, and ${ }^{3}$ Department of Physiology and Cellular Biophysics, \\ College of Physicians and Surgeons, Columbia University Medical Center, New York, New York, USA.
}

\begin{abstract}
Infection by Staphylococcus aureus strain USA300 causes tissue injury, multiorgan failure, and high mortality. However, the mechanisms by which the bacteria adhere to, then stabilize on, mucosal surfaces before causing injury remain unclear. We addressed these issues through the first real-time determinations of USA300-alveolar interactions in live lungs. We found that within minutes, inhaled USA300 established stable, self-associated microaggregates in niches at curved, but not at flat, regions of the alveolar wall. The microaggregates released $\alpha$-hemolysin toxin, causing localized alveolar injury, as indicated by epithelial dye loss, mitochondrial depolarization, and cytosolic $\mathrm{Ca}^{2+}$ increase. Spread of cytosolic $\mathrm{Ca}^{2+}$ through intercellular gap junctions to adjoining, uninfected alveoli caused pulmonary edema. Systemic pretreatment with vancomycin, a USA300-cidal antibiotic, failed to protect mice infected with inhaled WT USA300. However, vancomycin pretreatment markedly abrogated mortality in mice infected with mutant USA300 that lacked the aggregation-promoting factor PhnD. We interpret USA300-induced mortality as having resulted from rapid bacterial aggregation in alveolar niches. These findings indicate, for the first time to our knowledge, that alveolar microanatomy is critical in promoting the aggregation and, hence, in causing USA300-induced alveolar injury. We propose that in addition to antibiotics, strategies for bacterial disaggregation may constitute novel therapy against USA300-induced lung injury.
\end{abstract}

\section{Introduction}

When bacteria encounter host mucosal membranes, they must first adhere to the mucosal surface, stabilize by withstanding mucosal defense mechanisms, then induce pathogenic processes that lead to tissue injury. However, direct data in support of these hostbacterial interactions that critically underlie infective pathology remain unavailable. Thus, although alveolar injury due to lung infection is a major cause of mortality $(1,2)$, there is little understanding of how inhaled bacteria stabilize in alveoli to cause the injury. Here we consider this question in relation to the emergent, highly virulent Staphylococcus aureus strain USA300, which causes a form of rapidly occurring alveolar injury that associates with severe mortality (3-5).

It is understood from studies in cultured cells that alveolar stabilization of $S$. aureus might involve binding of bacterial surface proteins to host molecules, including cytokine receptors (6) and adhesive matrix molecules that recognize microbial surface components (7). Studies in vitro indicate that the bacteria may stabilize through biofilm formation $(8,9)$, charge interactions (10), or PhnD, the substrate-binding protein of the bacterial ATP-binding cassette (ABC) transporter for phosphonates (11). Once stabilized on the alveolar epithelium, $S$. aureus may cause injury by metalloproteinase activation (12), cytokine receptors (6), necroptosis (13), and mitochondrial dysfunction (14). However, direct evidence is not available that these proposed mechanisms apply to intact alveoli (15).

Conflict of interest: The authors have declared that no conflict of interest exists Submitted: July 5, 2017; Accepted: January 4, 2018.

Reference information: J Clin Invest. 2018;128(3):1074-1086.

https://doi.org/10.1172/JCI95823.
In this regard, it is important to consider the roles played by alveolar defense mechanisms that might diminish direct bacterial-epithelial contact and promote removal of inhaled particles. Thus, alveolar surfactant forms a protective liquid layer that inhibits bacterial contact with the epithelium (16). Phagocytosis by alveolar macrophages (17) and convective removal by the flow of alveolar wall liquid (AWL) (18) are likely to eliminate bacteria from alveoli. The extent to which these defense mechanisms impact alveolar stabilization of inhaled USA300 requires, we believe, real-time evidence of alveolar bacterial-epithelial interactions in alveoli.

We addressed these issues by means of optical imaging of live alveoli (19-21). Our goal was to determine bacterial and epithelial responses that follow early interactions between the alveolar wall and USA300, with an eye to understanding the time course of bacterial stabilization and subsequent epithelial injury. We also addressed the puzzling feature of this pathology that although USA300 might be antibiotic sensitive in vitro $(22,23)$, antibiotics tend to be ineffective in containing lung injury (23-25), possibly because of host factors.

We considered that inhaled bacteria might spread diffusely across the alveolar surface, causing damage at all sites of epithelial contact, or they might cluster, causing spatially extensive alveolar injury by spread of $\alpha$-hemolysin (Hla) from the clusters. However, our findings were opposite. Remarkably, USA300 rapidly formed impermeable microaggregates (MAs) in alveolar niches, accounting for antibiotic inefficacy. The MAs induced highly localized epithelial injury that spread through intercellular gap junctions (GJs), expanding lung injury, and causing high mortality. For the first time to our knowledge, these findings attest to the importance 
A

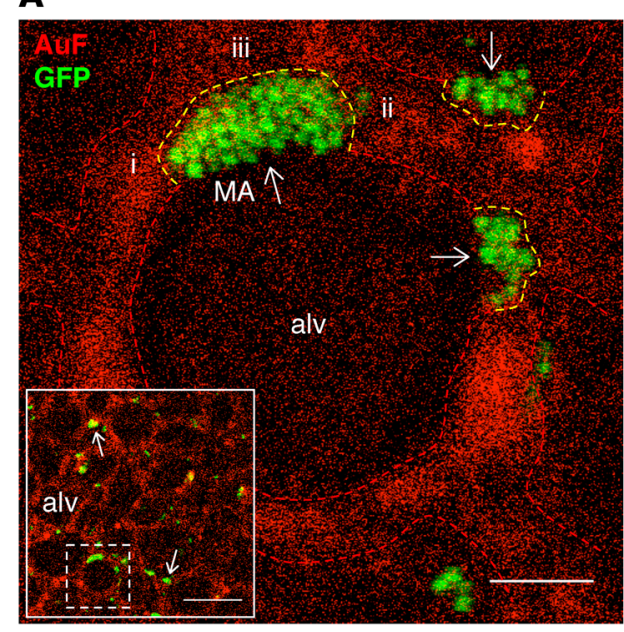

D

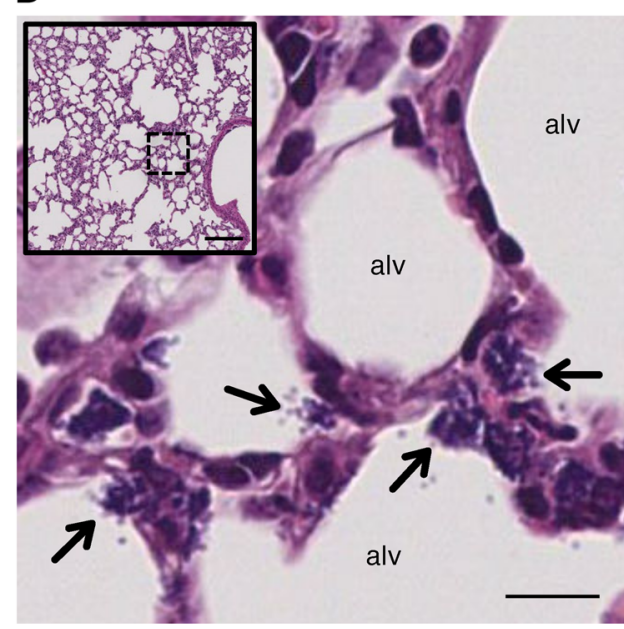

B

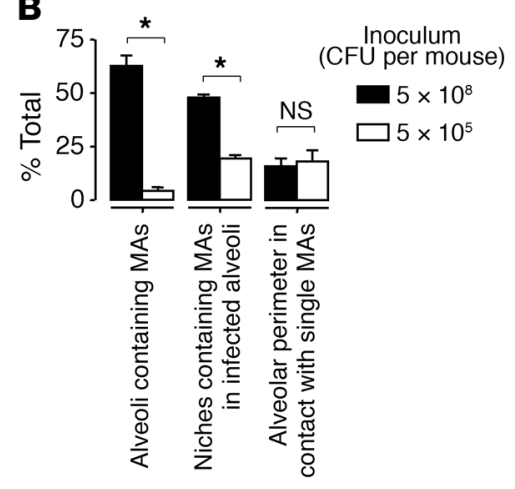

C

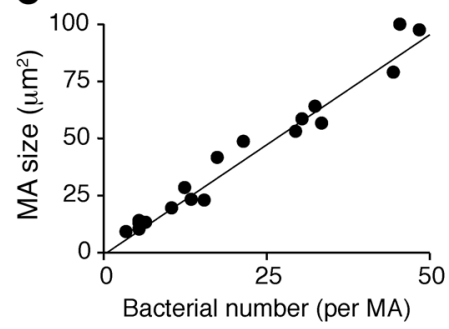

E

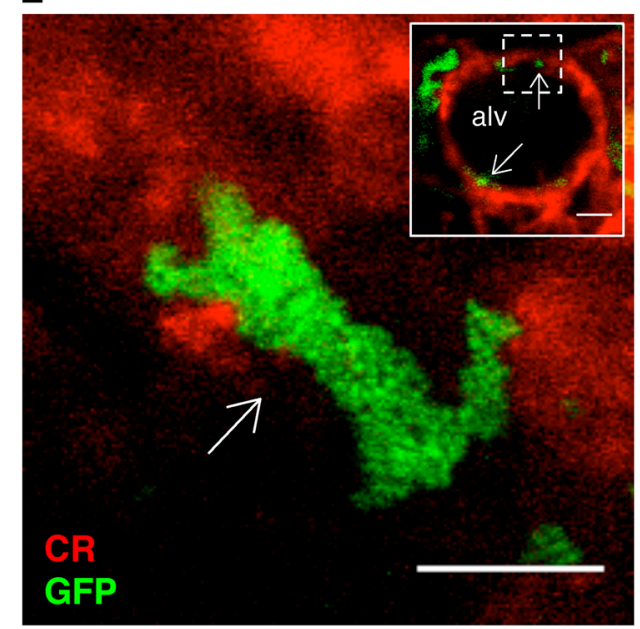

Figure 1. Inhaled S. aureus form MAs at alveolar niches. (A) Low-power (inset) and high-power confocal images of a live mouse alveolus (red) show different-sized MAs (arrows) of GFP-labeled S. aureus strain USA300 (green) at niches, curved alveolar regions where alveolar septa converge. Example niche-forming septa are indicated by i-iii. Dashed lines demarcate alveolar walls. AuF, autofluorescence; alv, alveolus. Scale bars: 50 (inset) and $8 \mu \mathrm{m}$. Replicated in 4 lungs. (B) Effect of inoculum size on MA distribution and distance of contact between MAs and the epithelium. Bars: mean \pm SEM; $n=3$ lungs in which fluorescence was quantified in 30 (left bars) or 3 (middle and right bars) alveoli per lung; ${ }^{*} P<0.05$ using 2-tailed $t$ test. (C) Bacterial counts of MAs. Each point represents a single MA selected from 3 lungs. Line calculated by linear regression $(P<0.05)$. (D) H\&E- and Gram-stained histological sections of lung tissue show low-power (inset) and high-power views of USA300 MAs (arrows) in niches of multiple alveoli. Scale bars: 100 (inset) and $10 \mu \mathrm{m}$. Replicated in 3 lungs. (E) Low-power (inset) and high-power confocal views of a calcein-loaded alveolus (red) in a human lung, 10 minutes after alveolar microinstillation of WT USA300 (green). Arrows indicate bacterial MAs in alveolar niches. CR, calcein red. Scale bars: 50 (inset) and $20 \mu \mathrm{m}$. Replicated in 3 alveoli.

of unique features of alveolar anatomy in bacterial pathobiology, in that alveolar niches provided sites of bacterial stabilization and alveolar GJs amplified the injury.

\section{Results}

USA300 rapidly form stable MAs in alveolar niches. Within 1 hour of

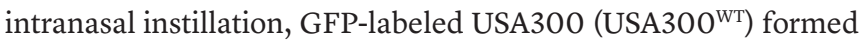
MAs on the alveolar epithelium at niches, curved regions of the alveolar wall at septal junctions (Figure 1A, arrows). MAs formed in more than $50 \%$ of alveoli viewed by confocal microscopy (Figure 1B). In infected alveoli, nearly half of the niches contained MAs (Figure 1B). Decreasing the bacterial number in the inhaled inoculum decreased the number of alveoli and niches containing MAs (Figure 1B). However, even with the lower bacterial inoc- ulum, MA size remained unchanged (Figure 1B), indicating that microanatomical features of alveoli determined MA size. MA size varied with numbers of bacteria contained in the MAs (Figure 1C). While all MAs made direct contact with the alveolar epithelium along the tissue-facing edge of the MAs, some MAs protruded into the alveolar lumen (Figure 1A). Together these findings indicate that the niches formed by the alveolar microanatomy determined bacterial clustering, thereby MA size.

To rule out the possibility that MA formation was restricted to subpleural alveoli, we fixed the lungs within 1 hour of intranasal instillation of bacteria. Histological sections revealed that MAs were in contact with alveolar epithelium in niches of alveoli located in central and peripheral lung regions (Figure 1D). These data affirm the confocal imaging findings and indicate that MA 
$5 \min$
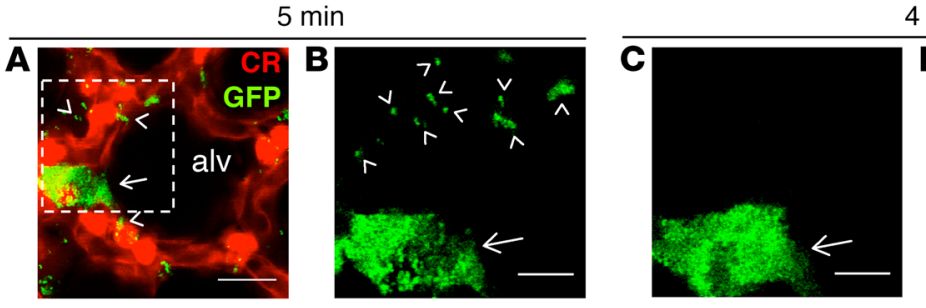

$\mathbf{E}$

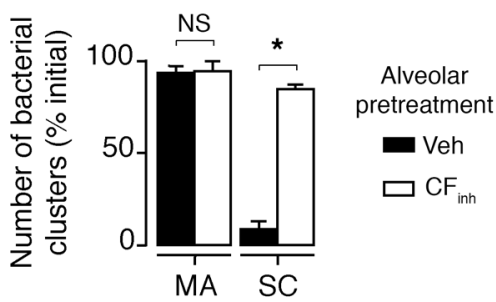

$\mathbf{F}$

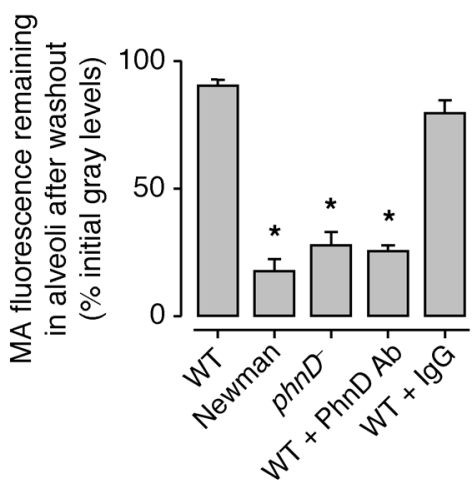

Figure 2. USA300 MAs are stable and solute impermeable. (A-E) Images (A-D) were obtained at the indicated times after bacterial instillation. A shows a vehicle-pretreated alveolus (alv), delineated by calcein red (CR) fluorescence in the epithelium (red), that contains an MA (arrow) of GFP-labeled WT USA300 (green) in an alveolar niche. Small clusters of 1-3 bacteria (arrowheads) are shown on the flat alveolar surface. $\mathbf{B}$ and $\mathbf{C}$ show the dashed region at high magnification. $\mathbf{D}$ is a high-magnification view from a different alveolus that was pretreated with CFTR inhibitor. Epithelial fluorescence was digitally removed in B-D. Note, small clusters were removed in $\mathbf{C}$ but not $\mathbf{D}$. Scale bars: 20 (A) and 5 (B-D) $\mu \mathrm{m}$. Bars (E) show group data for bacterial removal 4 hours after bacterial microinstillation. SC, small clusters; Veh, vehicle; $\mathrm{CF}_{\text {inh, }}$, CFTR inhibitor. Bars: mean $\pm \mathrm{SEM} ; n=3$ lungs (10 clusters quantified per lung); ${ }^{*} P<0.05$ as indicated using 2-tailed $t$ test. (F) Bars show fluorescence of the indicated microinstilled bacteria. Washout was given 30 minutes after each microinstillation. WT, WT USA300; phnD-, PhnD-deficient USA300. For fourth and fifth bars, WT was preincubated with the indicated antibodies. Bars: mean \pm SEM; $n=3$ lungs (5 MAs quantified per lung); ${ }^{*} P<0.05$ vs. left bar using ANOVA with Bonferroni correction. (C) Confocal images show epithelium (red) of 2 alveolar niches containing MAs (arrows) of microinstilled USA300 (green). USA300 are GFP-labeled WT (top) and calcein green-loaded (CG-loaded) phnD- (bottom). One hour after USA300 instillation, Alexa Fluor (AF; blue) was microinstilled in alveoli. In the right images, MA fluorescence was digitally removed and replaced by outlines. Open arrow highlights Alexa Fluor penetrance of the MA formed by phnD'. Scale bars: $8 \mu \mathrm{m}$. Replicated in 3 lungs.

formation in niches was a generalized phenomenon that occurred in alveoli throughout the lung.

To quantify the time course of MA formation, we microinstilled alveoli with USA300 ${ }^{\text {WT }}$ through glass micropipettes. Surprisingly, this procedure induced MA formation within minutes of microinstillation (Supplemental Figure 1A; supplemental material available online with this article; https://doi. org/10.1172/JCI95823DS1), attesting to the rapidity of the aggregation process. Since MA diameters exceeded micropipette tip diameters (Supplemental Figure 1, B and C), the MAs could not
$4 \mathrm{~h}$

have formed before microinjection and therefore could not have been directly injected. Moreover, MAs did not form in vitro (Supplemental Figure 1, B and C); hence they could not have been inserted into the micropipette before microinjection. MAs also formed within 10 minutes in human lungs (Figure $1 \mathrm{E})$, indicating that rapid MA formation in alveolar niches was not mouse specific. These findings indicate that irrespective of whether the bacteria were inhaled or directly microinstilled, MAs formed rapidly after USA300 ${ }^{\text {WT }}$ entered alveoli.

In contrast to alveolar niches, from which MAs were not spontaneously cleared (Figure 2, A-C, arrows), flat alveolar septa did not support MA formation. At these locations USA300 ${ }^{\text {WT }}$ formed small clusters of 1-3 bacteria (Figure 2, A-C, arrowheads), which cleared spontaneously. However, alveolar pretreatment with an inhibitor of the cystic fibrosis transmembrane conductance regulator (CFTR), $\mathrm{CFTR}_{\text {inh }}-172$, which inhibits AWL flow (18) but may have other nonspecific effects, blocked the spontaneous removal of small bacterial clusters (Figure 2, D and E), suggesting that the AWL washed out bacteria that did not incorporate into MAs. Taking these findings together, we conclude that localization of the bacteria to niches in curved alveolar segments was critical to the formation of stable MAs.

To further evaluate the stability of the USA300 ${ }^{\text {WT }}$ MAs, we gave multiple, prolonged microinstillations of buffer to MA-containing alveoli. However, these procedures failed to dislodge the MAs (Figure 2F, first bar), further attesting to their stability. To determine whether other bacteria and particles also induced stable MA formation, we gave alveolar microinstillations of fluorescent plastic beads (Supplemental Figure 1D), Pseudomonas aeruginosa, Klebsiella pneumoniae, or Newman, a different $S$. aureus strain. Although each of these instillations also induced the formation of MAs in alveolar niches, these MAs could be readily washed out by alveolar buffer microinstillations (Figure 2F, second bar, and Supplemental Figure 2A); hence they were not stable. These findings indicate that although nonspecific bacteria as well as inert particles clustered at alveolar niches, the MAs formed by USA $300^{\mathrm{WT}}$ alone were stable.

USA300 proteins determine stability of alveolar MAs. S. aureus aggregate in vitro through homophilic interactions between bac- 
A

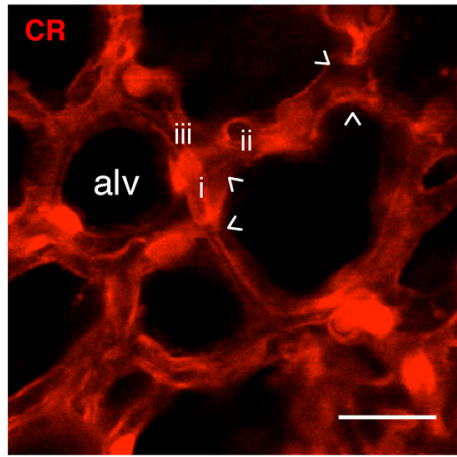

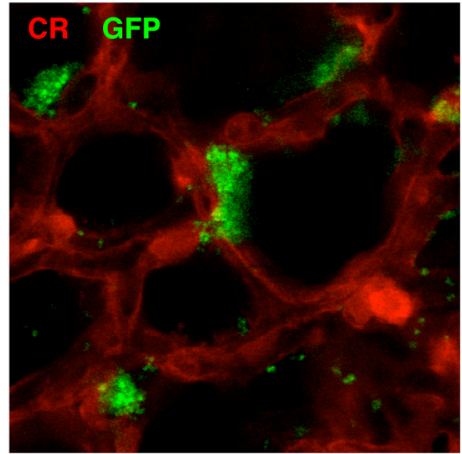

D

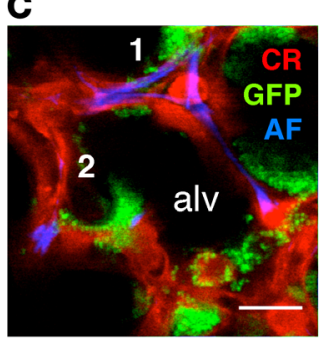

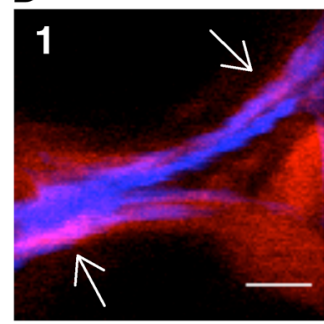

E

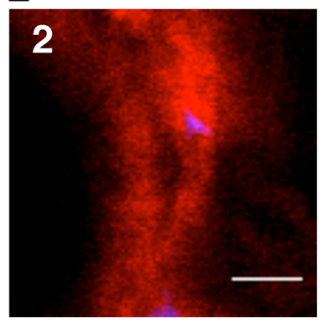

B

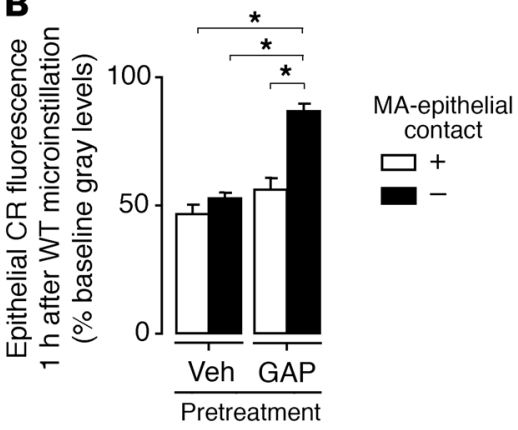

G

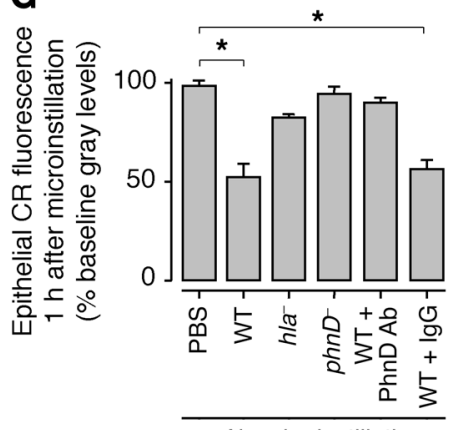

H

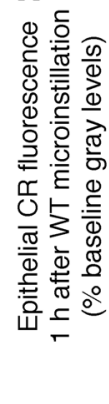

Alveolar instillation

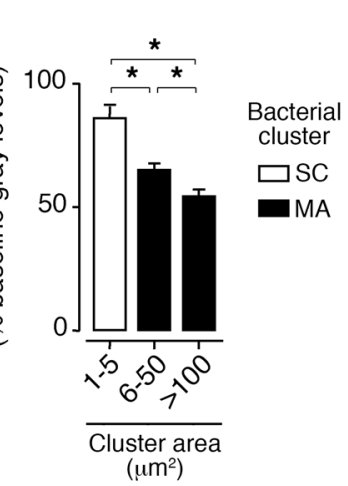

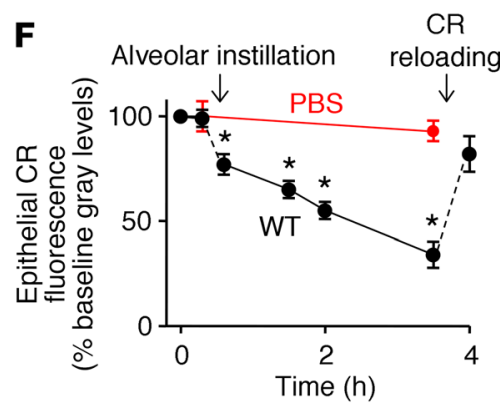

I

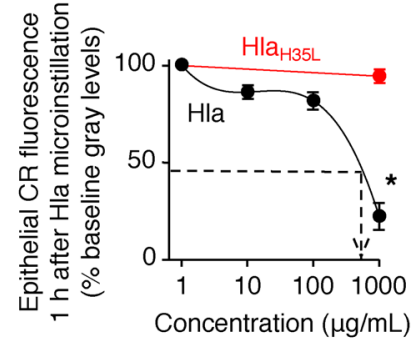

Figure 3. USA300 MAs cause localized epithelial injury. All microinstillations were in alveoli. (A) Confocal images show epithelial calcein red (CR) fluorescence (red), before (left) and 1 hour after (right) MA (green) formation by WT USA300 (GFP) in alveoli (alv). Example niches (arrowheads) and niche-forming septa (i-iii) are indicated. Scale bar: $20 \mu \mathrm{m}$. (B) Alveolar fluorescence at WT) MA-associated (+) and MA-free (-) sites. Alveoli were pretreated with vehicle (Veh) or GAP26/27 (CAP). Bars: $n=3$ lungs (4 sites quantified per lung). (C-E) Confocal images show alveoli (red) in which we induced WT MA (green) formation, then instilled Alexa Fluor (AF) dye (blue). MA fluorescence was digitally removed in high-magnification views of septa 1 (D) and 2 (E). Pink pseudocolor (arrows) signifies red-blue merge only for MA-associated epithelium (D). Scale bars: 15 (C) and 5 (D and $\mathbf{E}) \mu \mathrm{m}$. Replicated in 3 lungs. (F) Fluorescence loss after the indicated microinstillations. Points: $n=3$ lungs (30 alveoli quantified per lung). Arrows: time points of instillation and epithelial calcein reloading. (G) Fluorescence after the indicated microinstillations. hla and phnD', Hla- and PhnD-deficient USA300. Fifth and sixth bars: WT was preincubated with the indicated antibodies. Bars: $n=3$ lungs (30 alveoli quantified per lung). (H) Effect of WT cluster size on fluorescence at cluster-associated sites. SC, small clusters of 1-3 bacteria. Bars: $n=3$ lungs ( 3 sites quantified per lung). (I) Concentration dependence of Hla-induced calcein loss. Line calculated by polynomial regression $(P<0.05)$. Dashed arrow: Hla concentration corresponding to fluorescence loss induced by USA300 MAs. Hla ${ }_{335 L}$, mutant Hla. Points: $n=3$ lungs (30 alveoli quantified per lung). All points and bars: mean $\pm \mathrm{SEM}$; ${ }^{*} P<0.05$ as indicated $(\mathbf{B}, \mathbf{G}$, and $\mathbf{H}$ ) or vs. baseline $(\mathbf{F})$ or leftmost point (I) using ANOVA with Bonferroni correction.

terial surface proteins $(8,26)$. To evaluate bacterial proteins in MA formation, we considered the role of $\mathrm{PhnD}$. Although $\mathrm{PhnD}$ is a component of the bacterial phosphonate $\mathrm{ABC}$ transporter $(27,28)$, reports indicate that it may have also an adhesive function in vitro (11). Alveolar microinstillation of PhnD-deficient mutant USA300 $\left(p h n D^{-}\right)(29)$, or USA300 ${ }^{\mathrm{WT}}$ preincubated with a polyclonal antibody that inhibits PhnD binding (11), caused MA formation. However, in each case we could wash out the MAs with alveolar buffer microinstillation (Figure 2F, third and fourth bars from left), indicating that the clusters were not stable. Microinstillation of USA $300^{\mathrm{WT}}$ preincubated with an isotype-matched control antibody formed washout- resistant MAs (Figure 2F, fifth bar). These findings indicate that $\mathrm{PhnD}$-mediated interbacterial adhesion in alveolar niches was critical for formation of stable MAs of USA300.

It is proposed that $S$. aureus bind epithelial surfaces by ligating epithelial proteins such as $\beta_{1}$ integrins, or the cytokine receptor TNF receptor type 1 (TNFR1) $(6,30)$. To evaluate these possibilities, we microinstilled USA $300^{\mathrm{WT}}$ in alveoli pretreated with microinstillations of polyclonal anti- $\beta_{1}$ integrin antibody, or in alveoli of TNFR1-null mice. In each case, USA300 ${ }^{\text {WT }}$ formed stable MAs (data not shown). In addition, we eliminated all proteins from the alveolar surface by giving alveolar 


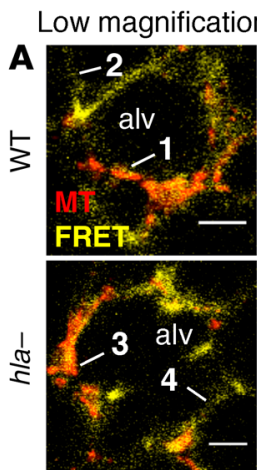

F

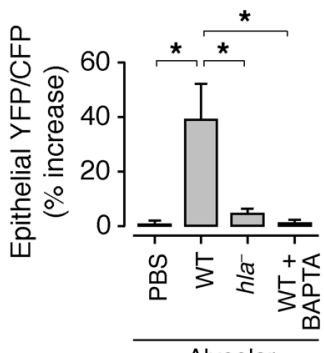

Alveolar instillation
$1 \mathrm{~h}$ after instillation
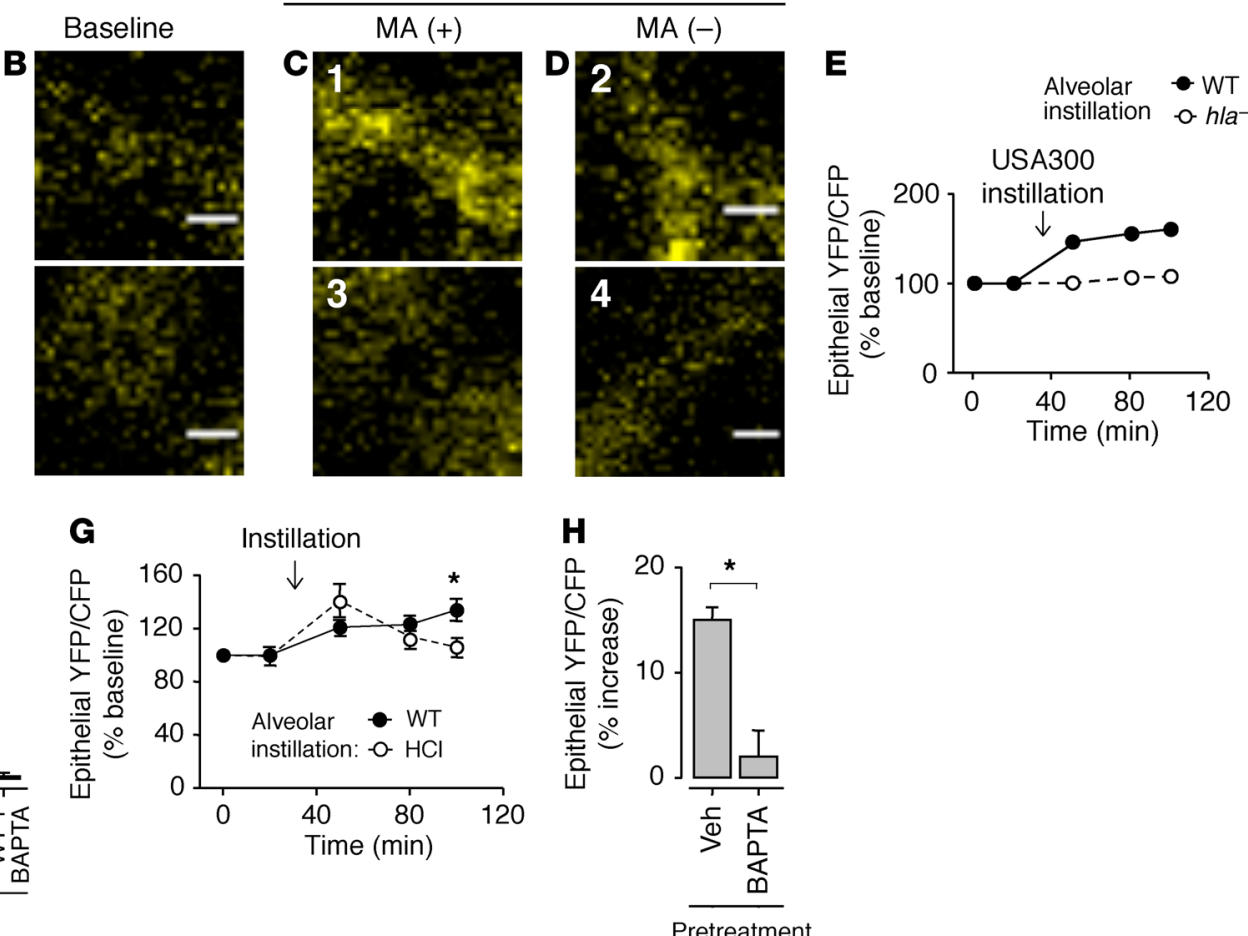

Figure 4. MA-induced cytCa ${ }^{2+}$ increase spreads from infected to uninfected alveoli. Images and quantifications are of alveolar fluorescence after epithelial transfection with the $\mathrm{Ca}^{2+}$-sensing YC2.3 FRET probe. CytCa ${ }^{2+}$ was measured in terms of the FRET-induced YFP/CFP fluorescence ratio. (A-D) Confocal images in A show epithelial (yellow) FRET-induced YFP (FRET) fluorescence of 2 representative alveoli (alv) containing MAs (red) of WT or Hla-deficient ( $h l a-$ ) USA300 as indicated. Bacteria are MitoTracker (MT) dye loaded. High-magnification images in B and C show MA-associated epithelial sites (1 and 3 in A), before (B) and 1 hour after (C) bacterial microinstillation. Images in $\mathbf{D}$ show high-magnification views of MA-free sites (2 and 4 in $\mathbf{A}$ ). Note, fluorescence increased at sites 1 and 2, but not 3 and 4. Bacterial fluorescence was digitally removed in C. Scale bars: 20 (A) and 5 (B and D) $\mu$ m. (E) Time course of FRET ratio increase at 2 representative MA-associated alveolar sites, after microinstillation of the indicated bacteria. Arrow indicates the time point of instillation. (F) Group data 1 hour after the indicated microinstillations. Right bar shows alveoli that were pretreated with BAPTA before WT instillation. Bars: mean \pm SEM; $n=4$ lungs ( 4 sites quantified per lung); ${ }^{*} P<0.05$ using ANOVA with Bonferroni correction. (G) Time-dependent effects of the indicated microinstillations. Arrow indicates the time point of instillation. $\mathrm{HCl}$, hydrochloric acid ( $\mathrm{pH} 1.0-1.2)$. Points: mean $\pm \mathrm{SEM} ; n=3$ lungs (3 sites quantified per lung); ${ }^{*} P<0.05$ vs. $\mathrm{HCl}$ using 2 -tailed $t$ test. (H) Group data 1 hour after alveolar WT microinstillation. Instilled alveoli were pretreated with vehicle (Veh) or BAPTA before instillation. Quantified alveoli did not contain bacteria. Bars: mean \pm SEM; $n=4$ lungs (4 sites quantified per lung); ${ }^{*} P<0.05$ using 2-tailed $t$ test.

microinstillations of trypsin. We confirmed that these microinstillations removed surface proteins as detected by biotin staining (Supplemental Figure 2, B-D). Microinstillation of USA300 ${ }^{\mathrm{WT}}$ in the protein-depleted alveoli also resulted in formation of washout-resistant MAs (Supplemental Figure 2, A, rightmost bar, and $\mathrm{D}$ ). Together, these findings suggest that MA formation and stabilization were not determined by alveolar epithelial surface proteins.

MAs inhibit penetration by an antibiotic-sized solute. Because of the above evidence indicating interbacterial adhesion in MAs, we considered that MAs may have diminished permeability to small solutes, an issue that might relate to antibiotic penetrance of these structures. Accordingly, in MA-containing alveoli we microinstilled fluorescent Alexa Fluor, a water-soluble solute of molecular weight $(1 \mathrm{kDa})$ similar to that of the antistaphylococcal antibiotic vancomycin. Alexa Fluor penetrance in USA300 ${ }^{\text {WT }}$ MAs was negligible (Figure $2 \mathrm{G}$, top row). By contrast, $p h n D^{-}$MAs were markedly penetrable by Alexa Fluor (Figure 2G, bottom row). We interpret that PhnD-mediated linkages on the bacterial wall of stabilized, microaggregated USA300 ${ }^{\mathrm{WT}}$ formed a barrier that blocked solute penetrance to the MA interior, whereas unstable MAs were freely permeable. Thus, stable USA300 MAs might hinder penetrance, hence efficacy of antibiotics.

USA30O causes rapid epithelial injury localized to MA sites. Findings in cultured cells indicate that staphylococci cause cell injury by Hla-induced membrane damage $(31,32)$. However, there is no understanding as to the rapidity of the membrane damage, or the role of staphylococcal aggregates in the process. Further, no direct data are available attesting to Hla-induced membrane damage in the intact alveolar epithelium, where membrane damage might be repaired spontaneously $(19,33)$. To test this hypothesis, we evaluated epithelial membrane damage at the MA-alveolar interface in terms of epithelial calcein fluorescence, which decreases following membrane damage (34). After alveolar instillation of USA300 ${ }^{\text {WT }}$, calcein fluorescence decreased in MA-associated epithelium (Figure 3, A and B, first bar from left), signifying epithelial membrane damage. Interestingly, calcein loss also occurred in MA-free epithelia (Figure 3, A and B, second bar from left). Hence, we considered that the MA-free response could be secondary to gap junctional effects. Accordingly, we treated 
Alveolar instillations
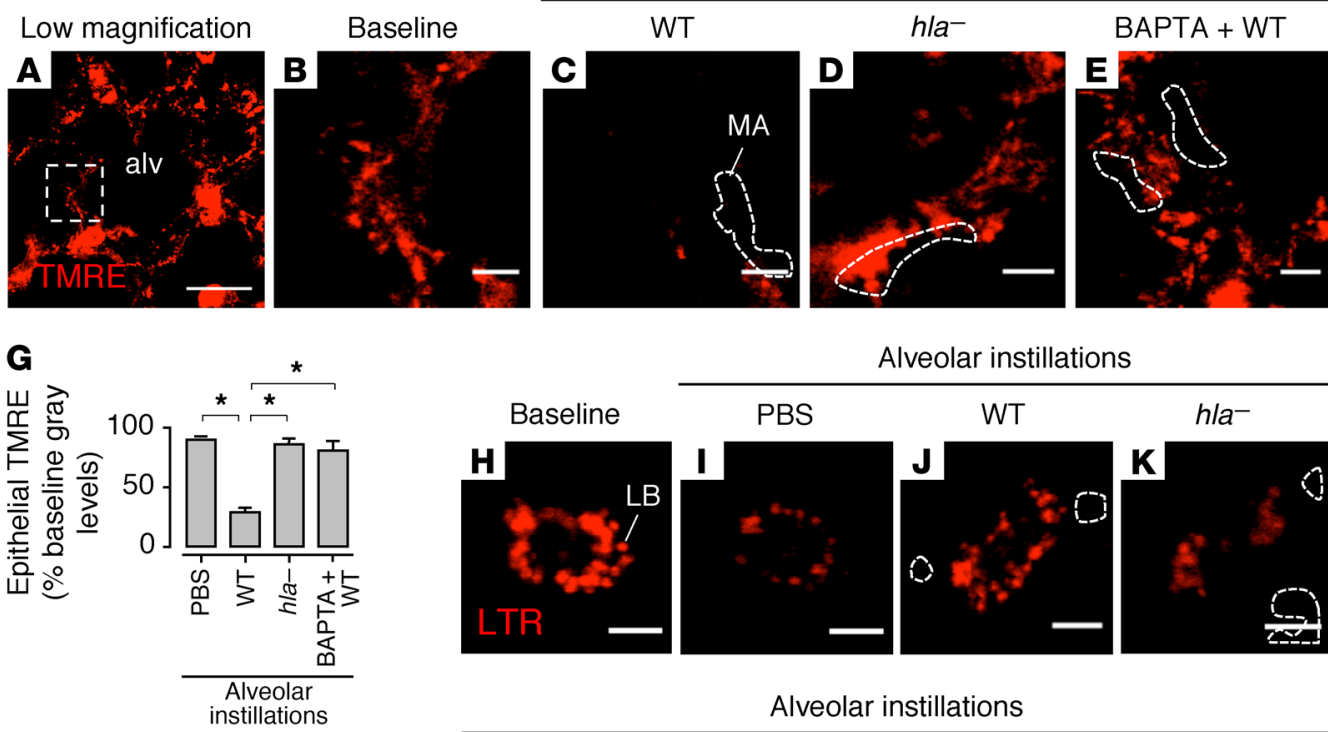

Alveolar instillations

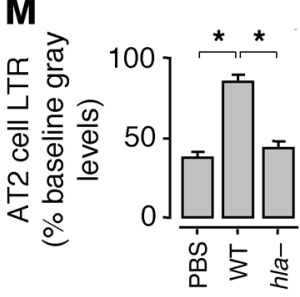

Alveolar
instillations

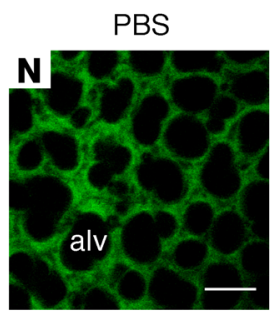

FD20

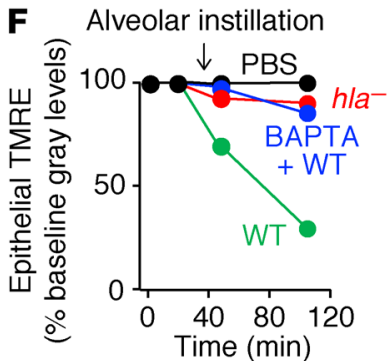

L Alveolar instillation, then inflation
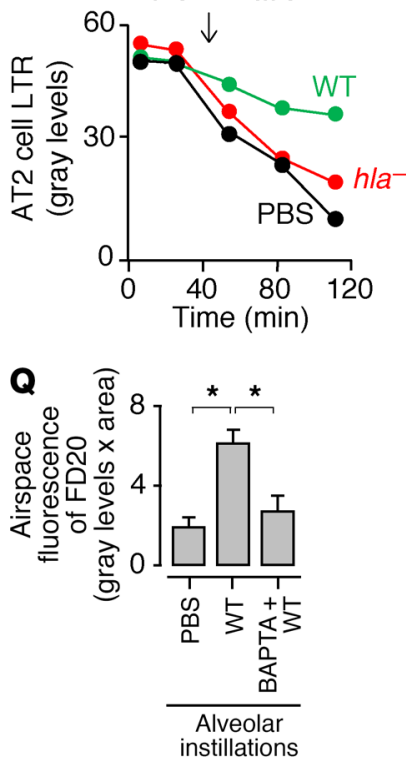

Figure 5. USA300 MAs rapidly induce Hla- and $\mathrm{Ca}^{2+}$-dependent loss of alveolar function. Confocal images and quantifications show fluorescence in live alveoli. All bars: mean $\pm \mathrm{SEM}$; ${ }^{*} P<0.05$ as indicated using ANOVA with Bonferroni correction. (A-C) A shows an alveolus (alv) with epithelial tetramethylrhodamine, ethyl ester (TMRE), fluorescence. B and C show the marked region (dashed rectangle) at high magnification, before (B) and 1 hour after (C) microinstillation of WT USA300 (WT). D and E are high-magnification regions of other alveoli that received Hla-deficient USA300 (hla ${ }^{-}$) or BAPTA pretreatment, respectively. Bacterial fluorescence was digitally removed in C-E. Dashed regions indicate MA locations. Scale bars: 30 (A) and 5 (B-E) $\mu \mathrm{m}$. Plots (F) and bars (C) show effects of the indicated treatments on fluorescence at MA-associated epithelial sites. Plots are representative for individual sites. Bars show group data 1 hour after the indicated microinstillations; $n=3$ lungs (3 sites quantified per lung). (H-M) H shows fluorescence of alveolar type 2 (AT2) cell lamellar bodies (LBs) loaded with LysoTracker Red (LTR). We microinstilled alveoli as indicated, transiently hyperinflated the lung, then obtained images I-K. LB fluorescence loss indicates hyperinflation-induced surfactant secretion. Bacterial fluorescence was digitally removed in J and $\mathbf{K}$. Dashed regions indicate MA locations. Scale bars: $5 \mu \mathrm{m}$. Plots (L) are representative for individual cells. Bars (M) show group data 1 hour after the indicated microinstillations; $n=3$ lungs ( 5 cells quantified per lung). ( $\mathbf{N}-\mathbf{Q}$ ) Alveoli were pretreated with vehicle ( $\mathbf{N}$ and $\mathbf{0}$ ) or BAPTA (P), then microinstilled as indicated. After 6 hours, we gave intravascular 20-kDa dextran (green), then obtained the images. MAs are indicated in red. Arrows indicate dextran-filled airspaces. FD20, FITC-labeled dextran. Scale bars: $50 \mu \mathrm{m}$. Bars (Q): $n=3$ lungs (10 alveoli quantified per lung).

alveoli with GAP26/27, an inhibitor of connexin 43-containing (Cx43-containing) GJs $(21,35)$. GAP26/27 pretreatment inhibited calcein loss at MA-free sites (Figure 3B, fourth bar from left), but not at MA-associated sites (Figure 3B, third bar from left), affirming a role for GJs in the effect and ruling out the possibility that membrane damage occurred at MA-free sites.

To further evaluate the localization of membrane damage at MA sites, we microinstilled alveoli with the dye Alexa Fluor, which is water soluble and membrane impermeable. Therefore, extracellular Alexa Fluor is not taken up in cells with intact membranes. Hence, we reasoned that appearance of Alexa Fluor fluorescence in the epithelial cytosol would indicate membrane damage in the fluorescent cell. Our findings indicate that following alveolar microinstillation, Alexa Fluor appeared in the epithelial cytosol, as evident by merged pseudocolor at MA-associated (Figure 3, C and D) but not at MA-free sites (Figure 3E). These findings affirm that membrane damage occurred only in MA-associated epithelia.

Although epithelial calcein fluorescence was stable after PBS microinstillation, the USA300 ${ }^{\mathrm{WT}}$-induced calcein loss initiated in minutes and continued for hours (Figure 3F). After the fluorescence decreased by more than $50 \%$, we reloaded calcein in the epithelium. This procedure returned cytosolic fluorescence to baseline (Figure 3F). Since the activity of cytosolic esterases determines calcein fluorescence (36), our ability to successfully reload calcein indicated that the epithelium was viable.

An Hla-deficient mutant of USA300 ( $\left.h l a^{-}\right)$that does not secrete Hla toxin (37) formed MAs (Supplemental Figure 2E) but failed to induce calcein loss (Figure 3G), affirming that Hla was critical for 
$\mathbf{A}$

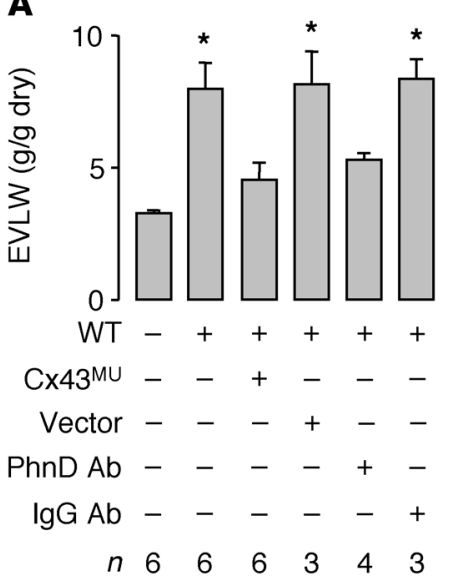

D

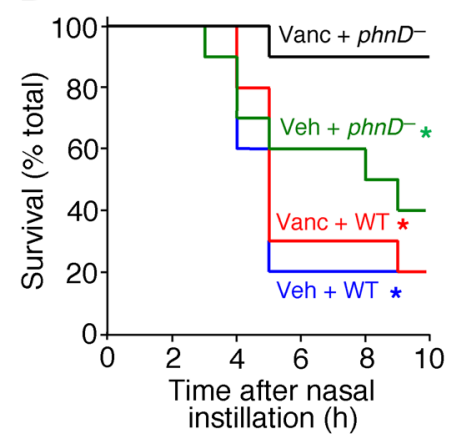

B

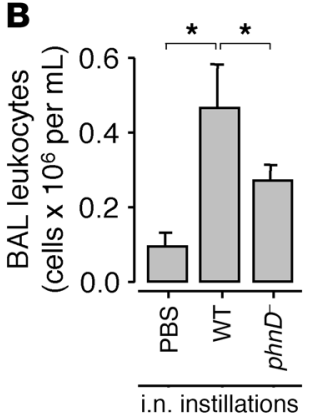

E

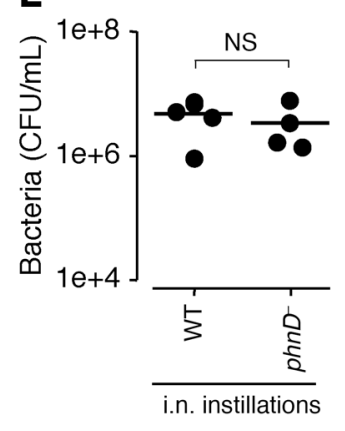

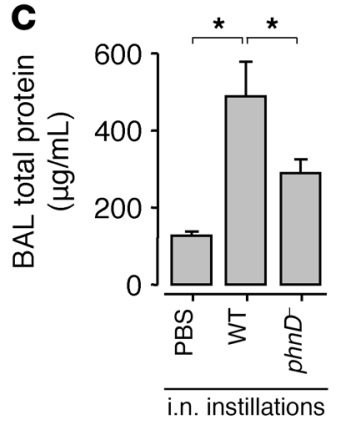

i.n. instillations

Figure 6. Inhibition of bacterial aggregation and G) function protects against USA300-induced lung injury. (A) Bars show blood-free extravascular lung water (EVLW) content in untreated mice, or 3 hours after intranasal instillation of WT USA300 (WT). For the third and fourth bars from left, mice were pretreated, 48 hours before WT instillation, with intranasal administration of liposome-complexed plasmid DNA encoding mutant connexin 43 protein ( $\left(x 43^{M U}\right)$ or empty vector (Vector). For the fifth and sixth bars from left, WT were preincubated in PhnD-blocking antibody or nonblocking IgC as indicated, then instilled. Bars: mean \pm SEM; $n$ as indicated; ${ }^{*} P<0.05 \mathrm{vs}$. left bar using ANOVA with Bonferroni correction. (B and C) Group data 4 hours after the indicated intranasal (i.n.) instillations. BAL, bronchoalveolar lavage; $p h n D^{-}$, PhnD-deficient USA300. Bars: mean $\pm S E M ; n=4$ mice per group; ${ }^{*} P<0.05$ as indicated using ANOVA with Bonferroni correction. (D) Plots show mouse survival after intranasal instillation of WT or phnD- USA300 as indicated. Mice were pretreated with subcutaneous injection of vehicle (Veh) or vancomycin (Vanc) as indicated, 1 hour before bacterial instillation. There was no mortality in untreated control mice (not shown). $n=10$ mice per group; ${ }^{*} P<0.05$ vs. black line at 10 hours after instillation using log-rank test. (E) Whole-lung bacterial count 4 hours after the indicated instillations. Each point represents a single mouse. Lines indicate means. Statistical significance $(P<0.05)$ was determined using 2-tailed $t$ test.

the membrane damage. The extent of calcein loss related directly to the extent of MA-epithelial contact (Figure 3H), affirming that bacteria at the MA-epithelial interface determined the injury. Unstable bacterial clusters that were formed in alveolar niches by $p h n D^{-}$, or by $\mathrm{USA} 0 \mathrm{O}^{\mathrm{WT}}$ that were preincubated with $\mathrm{PhnD}$-inhibiting antibody, but not with noninhibiting antibody, failed to induce calcein loss (Figure $3 G$ ), indicating that MA stability was critical. Together, our findings underline a novel feature of USA300-induced epithelial injury, namely that in alveoli, the injury is attributable to Hla-induced membrane damage that occurs locally only at sites of stable MAs.

These findings suggested that Hla secreted by MAs was restricted to the epithelial membrane directly associated with the MAs, and that there was no diffusion of Hla to MA-free alveolar regions. We suggest that at MA-associated sites, calcein escaped across the injured epithelial membrane, causing localized fluorescence loss (Supplemental Figure 3). Subsequently, intracellular calcein diffusion through GJs from noninjured epithelium to the adjacent injured epithelium resulted in fluorescence loss even at the noninjured, MA-free epithelium. Together, these findings indicated that Hla-induced membrane damage was localized to MA-associated epithelium, but that the injury initiated intercellular communication of cytosolic constituents.

We considered that Hla might achieve high concentrations in clefts (Supplemental Figure 1A, arrows) at the MA-epithelial interface. To determine membrane-damaging concentrations, we gave alveolar microinstillations of purified Hla. Our findings indicate that Hla, but not a mutant Hla that cannot damage membranes $(12,38)$, induced rapid, concentration-dependent calcein loss in alveolar epithelium (Figure 3I). We interpret from the response curve that an Hla concentration of more than $100 \mu \mathrm{g} / \mathrm{ml}$ (Figure 3I, dashed line) accounted for the calcein loss induced at MA sites (Figure 3B, first bar from left). Hence, we suggest that the Hla concentration at the MA-epithelial interface exceeded concentrations previously applied to induce membrane damage in cultured cells $(12,31,32,38)$, attesting to unique features of alveolar anatomy that may have augmented Hla toxicity.

Epithelial injury at MAs induces GJ communication of cytosolic constituents with noninjured epithelium. Understanding of S. aureusinduced effects on cytosolic $\mathrm{Ca}^{2+}\left(\mathrm{cytCa}^{2+}\right)$, a major determinant of alveolar injury (19), has been hampered by the tendency of soluble $\mathrm{Ca}^{2+}$-sensing dyes to leak across Hla-damaged cell membranes (32). Hence, to assess cytCa ${ }^{2+}$ responses, we transfected alveoli with the $\mathrm{Ca}^{2+}$-sensing FRET probe YC2.3 $(39,40)$. The probe was well expressed in alveolar epithelium (Supplemental Figure 4A). Excitation of cyan fluorescent protein (CFP) resulted in FRETinduced yellow fluorescent protein (YFP) fluorescence (Supplemental Figure 4, A and B), which was inhibited after we selectively photobleached CFP (Supplemental Figure 4B). We assessed the validity of the FRET-induced YFP/CFP fluorescence ratio as a $\mathrm{cytCa}^{2+}$ reporter through alveolar microinstillation of acid, which induces localized epithelial pore formation and cytCa ${ }^{2+}$ entry (19). Acid instillation increased the FRET ratio in epithelium (Supplemental Figure 4C). Moreover, alveolar pretreatment with the $\mathrm{Ca}^{2+}$ chelator BAPTA-AM abrogated the acid-induced FRET response (Supplemental Figure 4C). These findings affirmed that increases of the FRET ratio reflected increases in $\mathrm{cytCa}^{2+}$. 


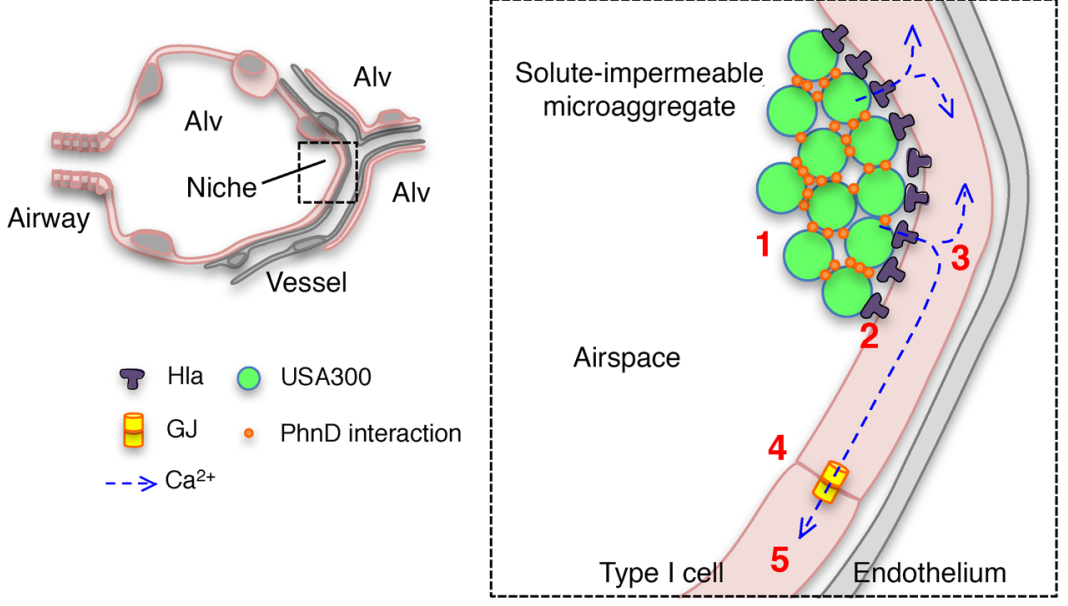

Figure 7. Mechanism of USA300-induced lung injury. Schematics show USA300 MA formation in an alveolar niche. Rapid interactions between USA300 and the alveolar epithelium initiate lung injury as follows: (1) Within minutes, inhaled USA300 stabilize in alveolar niches by forming solute-impermeable MAs through bacterial-bacterial, PhnD-mediated interactions. (2) Microaggregated bacteria rapidly release Hla toxin, leading to membrane damage in the localized MA-associated alveolar epithelial membrane. (3) Cytosolic $\mathrm{Ca}^{2+}$ increases in the MA-associated epithelium and (4) spreads to uninfected alveoli through connexin 43containing GJs, widely amplifying the spatial extent of the alveolar damage. (5) Subsequently, there is mitochondrial depolarization, surfactant secretion inhibition, and alveolar edema. Alv, alveolus.
Microinstillation of USA300 ${ }^{\mathrm{wT}}$, but not of $h \mathrm{la}^{-}$, increased epithelial cytCa ${ }^{2+}$ at MA sites (Figure 4, A-F). In contrast to the response to alveolar acid exposure that causes a transient cytCa ${ }^{2+}$ increase (19), the USA300 ${ }^{\mathrm{WT}}$-induced $\mathrm{cytCa}^{2+}$ increase did not return to baseline (Figure $4 \mathrm{G}$ ). Hence, in contrast to acid-induced membrane damage in which the membrane repairs spontaneously (19), there was no self-repair of the injured epithelial membrane at MA sites. Additionally, cytCa ${ }^{2+}$ increases occurred not only in alveoli containing MAs, but also in alveoli free of bacteria (Figure $4 \mathrm{H}$, left bar). Pretreatment of the infected alveoli with the intracellular $\mathrm{Ca}^{2+}$ chelator BAPTA-AM inhibited cytCa ${ }^{2+}$ increases at all locations (Figure 4, F and $\mathrm{H}$, right bar in each). Hence, $\mathrm{cytCa}^{2+}$ diffused from injured, MA-associated epithelium to epithelium of MA-free alveoli.

We considered that this spread of cytCa ${ }^{2+}$ could have injurious cellular effects as reflected by mitochondrial function (41), surfactant secretion (42), and alveolar barrier integrity (43). Within 15 minutes of MA formation, there was widespread loss of epithelial mitochondrial potential (Figure $5, A-G$ ) and inhibition of alveolar surfactant secretion (Figure $5, \mathrm{H}-\mathrm{M}$ ), which we quantified in terms of lamellar body exocytosis (44). After 6 hours, alveolar permeability to intravenously infused high-molecular weight dextran increased markedly (Figure 5, N-Q, Supplemental Figures 5 and 6, and Supplemental Video 1). These responses occurred in USA300 ${ }^{\text {WT }}$ infected and uninfected alveoli, but were absent in alveoli containing hla- MAs (Figure 5, D, F, G, and K-M), as well as in USA300 ${ }^{\text {WT }}$ containing alveoli that were pretreated with BAPTA-AM (Figure 5, E-G, P, and Q), or intranasally instilled with inoculums containing low bacterial counts (Supplemental Figure 5). We conclude that the increase of $\mathrm{cytCa}^{2+}$ in the MA-injured epithelium and its subsequent spread caused widespread alveolar dysfunction.

Bacterial disaggregation enhances antibiotic efficacy in USA30O lung infection. To determine whether the above cellular responses to USA300 ${ }^{\text {WT }}$ induced global lung responses, we quantified pulmonary edema formation in terms of lung extravascular water content (Figure 6A). Intranasal USA300 ${ }^{\mathrm{WT}}$ instillation increased extravascular lung water, indicating formation of pulmonary edema. This response was inhibited by alveolar expression of mutant $\mathrm{Cx} 43$ that blocks $\mathrm{Cx} 43$-containing GJs $(20,45)$. In addition, edema formation was blocked by instillation of USA300 ${ }^{\text {WT }}$ preincubated with PhnD-blocking antibody. These findings indicate that PhnD interactions in conjunction with GJ mechanisms accounted for USA300 ${ }^{\mathrm{WT}}$-induced pulmonary edema.

We considered survival responses in mice infected with USA300 given by intranasal instillation. Within 4 hours, USA300 ${ }^{\mathrm{WT}}$ infection increased the bronchoalveolar lavage (BAL) contents of leukocytes and total protein (Figure 6, B and C), resulting in $80 \%$ mortality (Figure 6D, blue line). However, despite the fact that lung bacterial counts were not different between USA300 ${ }^{\mathrm{WT}}$ - and $p h n D^{-}$-infected mice (Figure 6E), at 4 hours mice given $p h n D^{-}$bacteria had attenuated levels of BAL leukocytes and total protein (Figure 6, B and $\mathrm{C}$ ) as well a mortality rate of about $40 \%$, which was markedly less than that of USA300 ${ }^{\text {WT }}$ (Figure 6D, green line). Notably, the $p h n D^{-}$-infected mice continued to deteriorate and eventually had a mortality rate at 10 hours that was similar to that of USA300 ${ }^{\text {WT }}$ (Figure 6D). Thus, although phnD ${ }^{-}$ blocked stable MA formation, the bacteria may not have been eliminated, and could therefore have induced delayed mortality by undefined mechanisms.

To evaluate the effect of MA stability on antibiotic therapy, we treated mice with vancomycin, then instilled USA300. Vancomycin treatment failed to decrease USA300 ${ }^{\mathrm{WT}}$-induced mortality (Figure $6 \mathrm{D}$, red line). However, mortality in $p h n D^{-}$-infected mice was almost completely abrogated (Figure 6D, black line). In vitro studies indicated that there were no differences between USA300 ${ }^{\text {WT }}$ and $p h n D^{-}$colonies in regard to Hla secretion (Supplemental Figure 7, A and B), or vancomycin-induced inhibition of bacterial growth (Supplemental Figure 7C), ruling out toxin production and bacterial viability as factors in the survival response. Together, these findings indicate that although antibiotic pretreatment failed to protect against mortality due to USA300 ${ }^{\mathrm{WT}}$ infection, the pretreatment was highly effective against infection by mutant bacteria that failed to establish stable alveolar MAs (Figure 7).

\section{Discussion}

Our findings indicate that inhaled USA300 rapidly formed stable MAs in anatomical niches of alveolar walls. Bacteria that failed to locate at these niches failed to form MAs and were probably removed from alveoli by flow of the AWL. MA formation was critical to subsequent calcein loss from the epithelial cytosol, signi- 
fying damage to the epithelial membrane (34). Clustered bacteria that failed to form bacterial adhesive interactions, namely the PhnD-deficient mutant, did not induce epithelial injury. In confirmation of findings in cultured cells $(31,32)$, the membrane damage was Hla induced, since bacteria lacking Hla failed to cause calcein loss. However, the new understanding was that MA formation was critical to Hla-induced injury.

We expected the Hla secreted from MA sites to diffuse across the entire alveolar surface, causing widespread epithelial injury. However, 2 sets of findings failed to support this expectation. First, sites of membrane damage, which we detected in terms of epithelial internalization of the membrane-impermeable extracellular fluorophore Alexa Fluor, were localized to MA-apposed epithelia alone. Second, GJ inhibition blocked calcein loss in MA-free epithelia, indicating that with blockade of intercellular calcein diffusion there was no calcein loss, hence no membrane damage at epithelial sites free of MAs. These findings affirmed that injury was confined to the MA-epithelial interface and that Hla did not leave MAs in sufficient quantities to cause direct alveolar injury elsewhere. It is possible that a high concentration of accumulated Hla in cleft-like spaces at the MA-epithelial interface caused localized injury. To our knowledge, these findings are the first evidence that USA300 rapidly cause direct alveolar injury through MA formation in alveolar niches.

The present epithelial calcein loss is consistent with the notion that Hla causes epithelial injury through membrane damage (31, $32,46)$. The calcein loss initiated in minutes and was sustained for hours, indicating that the membrane damage did not undergo spontaneous repair (47). However, the fact that we could reload calcein in epithelia 4 hours after MA formation indicated that the membrane damage did not cause cell death in the alveolar epithelium. Nevertheless, $\mathrm{Ca}^{2+}$ entry occurred at sites of Hla-induced membrane damage, establishing sustained cytCa ${ }^{2+}$ increase. Since the alveolar epithelium communicates $\mathrm{Ca}^{2+}$ signals through GJs $(21,48)$, the cytCa ${ }^{2+}$ increases occurred not only in directly injured epithelia at MA sites, but also in MA-free epithelia of both infected and uninfected alveoli. Major consequences of the cytCa ${ }^{2+}$ increases were rapid induction of mitochondrial depolarization in the epithelium and loss of alveolar barrier properties. The intracellular $\mathrm{Ca}^{2+}$ chelator BAPTA-AM blocked both effects, pointing to the role of the epithelial cytCa ${ }^{2+}$ increase as the critical mechanism underlying USA300-induced alveolar injury.

Mitochondria are responsible for alveolar ATP production. MA formation rapidly blocked surfactant secretion, an ATP-dependent homeostatic function, presumably as a result of decreased ATP availability from depolarized mitochondria. Knockdown of alveolar Cx43, the major component of epithelial GJs, blocked pulmonary edema resulting from the loss of alveolar barrier properties. We conclude that the localized formation of nonrepairing membrane damage by Hla release at MA sites sustained epithelial $\mathrm{Ca}^{2+}$ influx for prolonged periods, leading to abrogation of essential aspects of alveolar function. The injury signals were rapidly communicated to uninfected alveolar regions through GJs, accounting for widespread lung injury.

Major new understanding regarding USA300 pathophysiology is that stabilization of inhaled bacteria was localized to specific anatomic regions of alveoli we termed niches. These niches occur in human and rodent lungs where alveolar septa converge, creating alveolar concavities that accumulate localized pools of alveolar liquid (49-51). With regard to clinical relevance, our data indicate that MA formation occurs in human lungs, suggesting that the bacteria may indeed localize at alveolar curvatures as infection initiates. However, subsequent events are difficult to interpret in the human context, since the available evidence comes largely from postmortem studies in which secondary responses are likely to mask niche localization. Further studies are required to clarify whether niche localization also occurs in lungs that might undergo loss of alveolar curvatures, as perhaps in emphysema. Notably, although USA300 delivered by nasal instillation or alveolar microinstillation reached all alveolar regions, we found no evidence of bacterial stabilization on flat septa. In fact, USA300 were apparently spontaneously removed from flat septa by the flow of AWL, consistent with the known ability of the AWL to wash out alveolar particles (18). Since trypsin elimination of alveolar surface protein ectodomains failed to inhibit MA formation, we interpret that alveolar surface proteins did not determine bacterial stabilization. Rather, irrespective of the numbers of bacteria instilled, the unique anatomy of alveolar curvatures rapidly localized the bacteria, setting the stage for interbacterial protein interactions, microaggregation, and alveolar damage.

A critical question relates to the mechanism of MA formation. Although our findings indicate that the niches are foci of particle accumulation, the micromechanics of particle flows that determine site-specific accumulation at alveolar curvatures have not been addressed. Since MA size determined the stability of the accumulated bacteria, we suggest that alveolar stabilization of USA300 resulted from a combination of alveolar microanatomy and MA size. Mechanical forces acting on bacteria in the niches may promote the formation of covalent bonds between adhesive proteins (52), stabilizing USA300 at these locations.

Although multiple bacterial proteins might be involved in the microaggregation, we identified a novel mechanistic role for PhnD. Whereas MAs formed by WT USA300 resisted washout despite our best efforts to dislodge them, genetic and immune inhibition of PhnD made them highly susceptible to washout, and we easily removed them from alveoli. We interpret that the MAs formed by PhnD-null USA300 were poorly organized and therefore unstable. Substrate-binding proteins of $\mathrm{ABC}$ transporters, such as $\mathrm{PhnD}(27,28)$, are known to play a role in bacterial adherence in vitro $(11,53,54)$. However, underlying adherence-inducing mechanisms remain unclear. The staphylococcal surface location of PhnD (11) may render PhnD accessible to interactions with surface proteins of juxtaposed bacteria. Since purified PhnD forms dimers in solution (27), we suggest that homophilic interactions between $\mathrm{PhnD}$ proteins rapidly induced interbacterial adhesion, leading to formation and stabilization of USA300 MAs.

Of therapeutic relevance, our findings indicate that antibiotic exclusion from bacterial clusters may contribute to failure of antibiotic therapy $(55,56)$. WT USA300 MAs excluded the Alexa Fluor dye, an antibiotic-sized solute, but PhnD-null MAs did not, suggesting that stabilized MAs of WT USA300 may diminish efficacy of antibiotics such as vancomycin that are USA300-cidal in vitro. To test this possibility, we determined survival in USA300-infected mice. Infection with WT USA300 caused rapid, severe mortality, suggesting that severe pulmonary edema induced hypoxia and, pos- 
sibly, massive cytokine release that caused cardiovascular collapse. These findings are consistent with clinical reports of rapid-onset septic shock and death in the setting of severe lung infection $(3,57$, 58). Notably, however, mortality due to PhnD-null infection was equally severe, if delayed, ruling out the possibility that the null bacteria were intrinsically less virulent. Although vancomycin pretreatment had no effect on mortality due to WT USA300, the pretreatment almost completely abrogated mortality due to PhnD-null bacteria. We interpret that the efficacy of vancomycin was attributable to poorly organized MAs formed by PhnD-null bacteria that enabled vancomycin penetration, hence bacterial killing. To our knowledge, these findings are the first to indicate that the manner in which bacteria initially organize against host epithelia, here as MAs, plays a critical role in the success of antibiotic therapy.

In conclusion, our findings indicate that interactions between inhaled USA300 and alveoli occur rapidly and are characterized first by localized bacterial stabilization and toxin-mediated injury, then by spread of injury signals through epithelial GJs. These events constitute a previously unrecognized role of the alveolar anatomy in establishing USA300-induced lung injury. The implications are that therapy for USA300 lung infection and injury must be targeted not only at the bacteria, but also at the alveolar response that included sustained increase of epithelial cytCa ${ }^{2+}$, depolarization of epithelial mitochondria, and deterioration of the alveolar barrier. New therapy might also address $G J$ inhibition as a strategy against USA300 lung infection. In addition, inhibition of the interbacterial adhesions that stabilize USA300 MAs may constitute novel treatment for combating failure of antibiotic therapy.

\section{Methods}

Fluorophores. We purchased Alexa Fluor 633 NHS ester $(50 \mu \mathrm{M})$, calcein AM $(5-10 \mu \mathrm{M})$, calcein red-orange AM $(10 \mu \mathrm{M})$, FITC-conjugated dextran ( $5 \mathrm{mg} / \mathrm{ml})$, LysoTracker Red (100 nM), MitoTracker Deep Red FM (MTDR; $5 \mu \mathrm{M}$ ), and tetramethylrhodamine, ethyl ester (TMRE; 10 $\mu \mathrm{M})$, from Life Technologies.

Reagents. We purchased trypsin-EDTA solution (0.25\%), EZ-Link Sulfo-NHS-SS-Biotin (1 mg/ml; lot 21331), and Alexa Fluor 633conjugated streptavidin $(1 \mu \mathrm{g} / \mathrm{ml})$ from Thermo Fisher Scientific; fluorescent plastic beads (Small Bead Calibration Kit) from Bangs Laboratories Inc.; CFTR $_{\text {inh }}-172(20 \mu \mathrm{M})$ from MilliporeSigma; GAP26 (200 $\mu \mathrm{M})$ and GAP27 $(500 \mu \mathrm{M})$ from Tocris; and 1,2-bis(o-aminophenoxy) ethane- $N, N, N^{\prime}, N^{\prime}$-tetraacetic acid tetra(acetoxymethyl ester) (BAPTA-AM; $100 \mu \mathrm{M}$ ) from Life Technologies. Purified Hla and purified Hla $_{\mathrm{H} 35 \mathrm{~L}}$ (gifts of Juliane Bubeck-Wardenburg's laboratory, University of Chicago, Chicago, Illinois, USA) were suspended in PBS with glycerol. For acid microinstillation, we titrated buffer with $\mathrm{HCl}$ to $\mathrm{pH}$ 1.0-1.2.

Solutions. We purchased PBS from Mediatech. Except where noted, agents were dissolved or suspended in PBS or a HEPES-buffered vehicle of pH 7.4 and osmolarity 295 mosm, containing $150 \mathrm{mM} \mathrm{Na}^{+}, 5$ $\mathrm{mM} \mathrm{K}^{+}, 1 \mathrm{mM} \mathrm{Ca}^{2+}, 1 \mathrm{mM} \mathrm{Mg}^{2+}$, and $10 \mathrm{mM}$ glucose.

Antibodies. We purchased antibodies against TNFR1 (catalog sc-7895) from Santa Cruz Biotechnology and against IgG1 (K isotype; catalog 51-4301) from eBioscience. Antibodies against HM $\beta 1-1 \quad\left(\beta_{1}\right.$ integrin; catalog 102201) (59) were purchased from BioLegend. Rabbit polyclonal antibodies against the $S$. aureus protein PhnD (11) (gift of Jeremy Yethon, Sanofi Pasteur, Cambridge, Massachusetts, USA) were generated and prepared by Sanofi Pasteur, using a method that included affinity purification with a PhnD column, dialysis into PBS, and concentration. Antibody-containing solutions were $4 \mu \mathrm{g} / \mathrm{ml}$ (TNFR1), $100 \mu \mathrm{g} / \mathrm{ml}$ (HMß1-1), and $8 \mu \mathrm{g} / \mathrm{ml}$ (PhnD, IgG1) in $\mathrm{Ca}^{2+}$ containing PBS (bacterial studies) or buffer (all others).

Bacterial strains. S. aureus strains included Newman, WT USA300 LAC (USA300 ${ }^{\mathrm{WT}}$ ), hla-deficient mutant USA300 LAC ( $h l a^{-}$) (gift of Juliane Bubeck-Wardenburg's laboratory, University of Chicago), and mutant USA300 LAC containing a transposon interrupting phnD, the gene for the phosphonate $\mathrm{ABC}$ transporter phosphonate-binding protein (strain NE1646, accession no. SAUSA300_0145; phnD-). The transposon-containing mutant was provided by the Network on Antimicrobial Resistance in Staphylococcus aureus (NARSA) and distributed by BEI Resources (29). Other bacteria included the P. aeruginosa (PA) strain PAK, the K. pneumoniae (KP) strain KP35, and, for hemolysis assessment, the $S$. aureus strain RN4220.

Bacterial preparation. We propagated $S$. aureus, $\mathrm{PA}$, and $\mathrm{KP}$ in $\mathrm{LB}$ broth (MP Biomedicals) to optical density (OD) 1 (S. aureus) or 0.5 (PA and $\mathrm{KP}$ ) at $600 \mathrm{~nm}$ (SPECTRAmax Plus, Molecular Devices). We grew single bacterial colonies overnight in a shaking incubator at $37^{\circ} \mathrm{C}$ and 200 rpm (Innova42, New Brunswick Scientific). On the day of experiments, we added 200- or 500- $\mu$ l culture aliquots to 10 or $50 \mathrm{ml}$ fresh $\mathrm{LB}$, respectively, and propagated the bacteria in a shaking incubator to the appropriate OD. For fluorescence imaging, we used GFP-labeled bacteria (PA, KP, and USA300 ${ }^{\mathrm{WT}}$ ) or bacteria that were incubated 5 minutes in calcein AM or MTDR ( $5 \mu \mathrm{M}$ each), then washed with PBS. Major experiments using GFP-labeled USA300 were replicated using non-GFP-labeled USA300 LAC. Bacteria containing GFP plasmids were grown in the presence of the selective antibiotic carbenicillin $(\mathrm{PA}, 300 \mu \mathrm{g} / \mathrm{ml})$, tetracycline $(\mathrm{KP}, 10 \mu \mathrm{g} / \mathrm{ml})$, or chloramphenicol $(S$. aureus, $10 \mu \mathrm{g} / \mathrm{ml}$ ). We incubated bacteria in antibody-containing solution for 5 minutes prior to lung instillation for selected experiments.

Bacterial instillation. Bacterial culture aliquots were centrifuged and resuspended in PBS containing $\mathrm{Ca}^{2+}$ and $\mathrm{Mg}^{2+}$. For intranasal instillation for imaging and extravascular lung water, we resuspended $10 \mathrm{ml}$ of culture $\left(\mathrm{OD}_{600 \mathrm{~nm}}=1\right)$ in $100 \mu \mathrm{PBS}$, then administered $50 \mu \mathrm{l}$ suspension to deliver $4 \times 10^{8}$ to $6 \times 10^{8} \mathrm{CFU}$ per mouse. For selected experiments, we resuspended $10 \mu \mathrm{l}$ of culture in $100 \mu \mathrm{l}$ PBS, then administered $50 \mu \mathrm{l}$ suspension to deliver $5 \times 10^{5} \mathrm{CFU}$ per mouse. For survival experiments, BAL studies, and bacterial counts, we resuspended $10 \mathrm{ml}$ of culture in $500 \mu \mathrm{l} \mathrm{PBS}$, then administered $50 \mu \mathrm{l}$ suspension to deliver $8 \times 10^{7}$ to $1 \times 10^{8} \mathrm{CFU}$ per mouse. For micropipette instillation in alveoli or onto a coverslip, we resuspended $1.5 \mathrm{ml}$ of culture in $150 \mu \mathrm{lBS}$, then administered approximately $10^{4} \mathrm{CFU}$ in $3 \mathrm{sec}-$ onds of discontinuous microinstillation (60). Detail regarding alveolar microinstillation methodology is provided below. PA and KP were prepared and instilled as described for $S$. aureus.

Animals. Animals were 5-10 weeks old and age-matched. All mice were male Swiss Webster (Taconic Biosciences Inc.) except for TNFR1null mice, which were male on a C57BL/6 background (Jackson Laboratory; C57BL/6-Tnfrsf1 $a^{\text {tmIImx }}$ ). For mouse anesthesia for intranasal instillations and surgical procedures, we gave inhaled isoflurane (4\%) and i.p. injections of ketamine (up to $100 \mathrm{mg} / \mathrm{kg}$ ) and xylazine (up to $5 \mathrm{mg} / \mathrm{kg}$ ).

Isolated, blood-perfused lungs. By our reported methods (18-21, 42,60 ), we excised intact lungs from anesthetized mice and perfused them with autologous blood via cannulas in the pulmonary artery and left atrium of the heart. We maintained pulmonary arterial and left atrial pressures of 10 and $3 \mathrm{~cm} \mathrm{H}_{2} \mathrm{O}$, respectively, and constant per- 
fusion flow rate $0.4-0.6 \mathrm{ml} / \mathrm{min}$ at $37^{\circ} \mathrm{C}$. We inflated the lungs with a gas mixture $\left(30 \% \mathrm{O}_{2}, 6 \% \mathrm{CO}_{2}\right.$, balance $\left.\mathrm{N}_{2}\right)$ through a tracheal cannula and maintained constant airway pressure of $5 \mathrm{~cm} \mathrm{H}_{2} \mathrm{O}$ using a continuous positive airway pressure machine. We diluted blood with $4 \%$ dextran (70 kDa; TCI America), 1\% FBS (HyClone, Thermo Fisher Scientific), and HEPES-buffered vehicle. Blood-buffer solution was pH 7.4 and osmolality 320 mosm $/ \mathrm{kg}$.

Alveolar microinstillation. We used hand-beveled glass micropipettes (tip diameter $6 \mu \mathrm{m}$ ) to micropuncture single alveoli under bright-field microscopy as we have previously reported (18-21, 42, 60). By way of the micropipettes, we microinstilled the micropunctured alveoli with fluorophores, reagents, solutions, and antibodies, resulting in their spread from the micropunctured alveolus to at least 20 neighboring alveoli within local lung acini, as evident by transient loss of optical discrimination between alveolar walls and air spaces. Return of optical discrimination occurred within seconds of each microinstillation, indicating free fluid rapidly drained from the alveolar lumens (61). We imaged the microinstilled alveolar fields but excluded the micropunctured alveoli from the region imaged. We microinstilled alveoli at 1 to 3 alveolar sites bordering each imaging field.

Live lung imaging and analysis. We viewed alveoli by confocal microscopy (LSM 510 META, Zeiss) with a $\times 40$ microscope immersion objective (Zeiss; numerical aperture 0.8). All images were acquired as single images using LSM Image Browser software. Images were recorded as $Z$-sections, 6-8 $\mu \mathrm{m}$ below the pleura. Optical thickness was set at $3 \mu \mathrm{m}$ for all imaging except FRET determinations, for which optical thickness was $13 \mu \mathrm{m}$. In experiments in which we microinstilled alveoli with multiple materials of different excitation spectra, we confirmed absence of bleed-through between fluorescence emission channels. We analyzed images using commercial software, Image (version 1.47, NIH). Brightness and contrast adjustments were applied to individual color channels of entire images and equally to all experiment groups. We did not apply further downstream processing or averaging.

Surfactant secretion induction. We induced surfactant secretion in isolated, blood-perfused lungs (IPLs) by a single hyperinflation, in which airway pressure was increased from 5 to $15 \mathrm{~cm} \mathrm{H}_{2} \mathrm{O}$ for 15 seconds (44).

Alveolar permeability determination. To determine alveolar barrier properties, we added FITC-dextran (20 kDa; $5 \mathrm{mg} / \mathrm{ml}$; FD20) to the IPL perfusate solution as we have previously reported (19). Alveolar permeability was detected by the presence of FD20 in alveolar airspaces. To quantify alveolar edema, we measured FD20 gray levels and fluorescence area in the 10 most affected alveolar airspaces per imaging field.

Lung tissue histology. After intranasal instillation of bacteria, we anesthetized mice and exsanguinated them by cardiac puncture, then placed cannulas in the trachea, pulmonary artery, and left atrium by way of median sternotomy. We excised the lungs and heart, inflated the lungs to $10 \mathrm{~cm} \mathrm{H}_{2} \mathrm{O}$, then perfused the lungs with $5 \mathrm{ml}$ PBS fol-

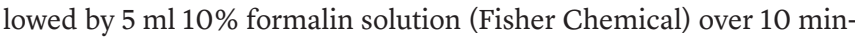
utes. We immersed the inflated lungs in $10 \%$ formalin solution for 24 hours, then rinsed and stored them in $70 \%$ ethanol before sectioning. Histological sections ( $3 \mu \mathrm{m}$ thickness) and staining were performed by the Columbia University Medical Center Molecular Pathology Core at the Herbert Irving Comprehensive Cancer Center.

Plasmid preparation and transfection. We chemically transformed DH5 $\alpha$ E. coli with plasmid DNA, then amplified and purified the plasmid using an EndoFree Plasmid Maxi Kit (Qiagen). Using our established methods $(19,20)$, we complexed plasmid DNA for, separately,
$\mathrm{Cx} 43^{\mathrm{Mu}}(2.05 \mu \mathrm{g} / \mu \mathrm{l})(20,45), \mathrm{YC} 2.3(2.13 \mu \mathrm{g} / \mu \mathrm{l}$; gift of Roger Tsien, UCSD, La Jolla, California, USA) (39), or empty vector, with freshly extruded unilamellar liposomes $(20 \mu \mathrm{g} / \mu \mathrm{l} ; 100 \mathrm{~nm}$ pore size; DOTAP, Avanti Lipids) in sterile Opti-MEM (Invitrogen). We administered up to $100 \mu \mathrm{g}$ plasmid DNA per mouse by intranasal instillation. Imaging and lung water experiments were carried out 48 hours after transfection.

$\mathrm{Ca}^{2+}$ determinations. We transfected mice with YC2.3 (39) DNA (gift of Roger Tsien's laboratory, UCSD) and viewed fluorescence in 3 channels: (a) cyan fluorescent protein (CFP) fluorescence (excitation $458 \mathrm{~nm}$, emission collection filter 475-505 nm), (b) yellow fluorescent protein (YFP) fluorescence (excitation $514 \mathrm{~nm}$, filter 530-600 nm), and (c) CFP-induced YFP (FRET) fluorescence (excitation $458 \mathrm{~nm}$, filter 530-600 nm). We affirmed baseline fluorescence by selective photobleaching. To quantify cytCa ${ }^{2+}$, we measured the ratio of FRET-induced YFP/CFP fluorescence in alveolar segments.

Extravascular lung water and lung blood content. We determined blood-free lung water content by the method of Selinger and colleagues (62), which we have used previously $(63,64)$. We excised mouse lungs from anesthetized mice in the absence of treatment or 3 hours after intranasal instillation of bacteria. We corrected the wet/dry ratio of the lung homogenate for blood water content. Hemoglobin standards and Drabkin's solution used to determine blood hemoglobin content were purchased from Stanbio and Sigma-Aldrich, respectively.

Survival assessment. Mice were treated 1 hour before intranasal USA300 instillation with subcutaneous injection $(65,66)$ of either vancomycin (15 mg/kg; lot SLBK2559V, Sigma-Aldrich) in a solution of $5 \%$ dextrose in sterile water (D5W) or an equivalent volume of D5W control solution. The mice were evaluated at frequent intervals following USA300 instillation, in accordance with the Animal Care Protocol approved for use in our laboratory. At each evaluation, the animals were assessed by a blinded investigator and either allowed to continue the survival study or selected for euthanization on the basis of an objective scoring system, which we have used previously $(20,21)$, that incorporates measures of weight, activity, grooming, and respiration.

Protein and leukocyte determinations on BAL fluid. We lavaged the lungs of anesthetized mice with ice-cold $\mathrm{Ca}^{2+}$-free PBS ( $1 \mathrm{ml} 5$ times) via a tracheal cannula. For total protein determinations, we centrifuged the first $1 \mathrm{ml}$ of BAL for 10 minutes at $400 \mathrm{~g}$ and $4^{\circ} \mathrm{C}$, then centrifuged the supernatant again for 20 minutes at $16,000 \mathrm{~g}$ and $4^{\circ} \mathrm{C}$. Total protein content was quantified using a plate reader (SPECTRAmax Plus, Molecular Devices), BSA standards ( $2 \mathrm{mg} / \mathrm{ml}$ in $0.9 \%$ saline; Thermo Fisher Scientific), and the Pierce PCA Protein Assay Kit (Thermo Fisher Scientific). For leukocyte determinations, BAL samples were pooled on a per-mouse basis and centrifuged for 10 minutes at $500 \mathrm{~g}$ and $4^{\circ} \mathrm{C}$. Resuspended pellets were incubated for 10 minutes in Türk's solution (Sigma-Aldrich) before manual counting of cells on a hemacytometer (Hausser Scientific).

Whole-lung bacterial count. We used sterile surgical instruments to excise lung lobes from anesthetized mice and immediately stored them in specimen bags in $-80^{\circ} \mathrm{C}$ freezer overnight. The following day, we homogenized the organs mechanically within the specimen bags and added $1 \mathrm{ml}$ sterile PBS. We centrifuged the homogenates for 5 minutes at $400 \mathrm{~g}$, then determined bacterial counts by serial dilutions on LB agar plates.

Human lungs. Human lungs declined for transplantation (gift of Michael Matthay, UCSF, San Francisco, California, USA) were shipped overnight in refrigerated containers, ensuring their stability and viability for 3 days (67). We inflated the right middle lobe with a gas mix- 
ture $\left(30 \% \mathrm{O}_{2}, 6 \% \mathrm{CO}_{2}\right.$, balance $\left.\mathrm{N}_{2}\right)$ through a bronchial cannula and maintained constant airway pressure of $10 \mathrm{~cm} \mathrm{H}_{2} \mathrm{O}$ using a continuous positive airway pressure machine. We micropunctured single alveoli as described above to instill solutions containing fluorophores or bacteria. Human alveoli were imaged as described for those of mice.

Hemolytic activity assay. Single colonies of bacteria were crossstreaked onto $5 \%$ sheep blood agar plates (Becton Dickinson) perpendicularly to the $\beta$-toxin-producing $S$. aureus strain RN4220, then incubated for 18 hours at $37^{\circ} \mathrm{C}(68)$. All USA300 strains except hla demonstrated a hemolysis pattern typical of Hla (68). We quantified Hla activity using a semiquantitative approach (69).

Antibiotic susceptibility assay. Overnight cultures were prepared from single bacterial colonies as described above. We simultaneously inoculated $5 \mathrm{ml} \mathrm{LB}$ broth aliquots containing various concentrations of vancomycin (lot SLBK2559V, Sigma-Aldrich) with $100 \mu$ l of the overnight culture. The cultures were incubated on a shaking incubator for 5 hours at $37^{\circ} \mathrm{C}$ and $200 \mathrm{rpm}$ (Innova42, New Brunswick Scientific). Vancomycin susceptibility was determined by turbidity assays obtained using spectrophotometry at $600 \mathrm{~nm}$ (SPECTRAmax Plus, Molecular Devices).

Statistics. All major groups comprised a minimum of 3 mice each. Numbers of mice per group and numbers of quantifications per mouse lung are indicated in the figure legends. Mean and SEM were calculated on a per-lung basis. We analyzed paired comparisons using the 2-tailed $t$ test and multiple comparisons using ANOVA with the Bonferroni correction. Survival comparisons were analyzed by the logrank test. We considered statistical significance at $P$ less than 0.05 .

Experimental design. Animals were selected randomly for experiments from the animal supply. Investigator blinding to group alloca- tion or experimental outcome was not performed, except as indicated for survival assessment.

Study approval. The Institutional Animal Care and Use Committee of Columbia University Medical Center approved all animal procedures. Human lungs were obtained in accordance with policies at the UCSF Medical Center.

\section{Author contributions}

JLH designed and carried out the experiments, prepared the figures, and wrote the initial manuscript. MNI assisted with FRET, mitochondria, and alveolar type 2 cell imaging experiments. MNI, DP, ASP, and SB contributed to the experimental design. JB was responsible for the overall project and wrote the initial manuscript. All authors edited the manuscript.

\section{Acknowledgments}

We thank A.C. Uhlemann, S. Sullivan, and S. Khan (Columbia University Medical Center) for assistance with the hemolytic activity assay. This work was supported by an American Heart Association Fellow-to-Faculty Transition Award, a Stony Wold-Herbert Fund Inc. Fellowship, and T32 Research Training Fellowship HL105323 to JLH and grants HL36024 and HL122730 to JB from the NIH.

Address correspondence to: Jahar Bhattacharya, Department of Medicine, 630 W. 168th Street, Room BB 8-812, New York, New York 10032, USA. Phone: 212.305.7093; Email: jb39@cumc. columbia.edu.
1. Rubenfeld GD, et al. Incidence and outcomes of acute lung injury. $N$ Engl J Med. 2005;353(16):1685-1693.

2. Damluji A, et al. Short-term mortality prediction for acute lung injury patients: external validation of the Acute Respiratory Distress Syndrome Network prediction model. Crit Care Med. 2011;39(5):1023-1028.

3. Hidron AI, Low CE, Honig EG, Blumberg HM. Emergence of community-acquired meticillinresistant Staphylococcus aureus strain USA300 as a cause of necrotising community-onset pneumonia. Lancet Infect Dis. 2009;9(6):384-392.

4. Carrillo-Marquez MA, Hulten KG, Hammerman W, Lamberth L, Mason EO, Kaplan SL. Staphylococcus aureus pneumonia in children in the era of community-acquired methicillin-resistance at Texas Children's Hospital. Pediatr Infect Dis J. 2011;30(7):545-550.

5 . Montgomery CP, et al. Comparison of virulence in community-associated methicillin-resistant Staphylococcus aureus pulsotypes USA300 and USA400 in a rat model of pneumonia. J Infect Dis. 2008;198(4):561-570.

6. Gómez MI, et al. Staphylococcus aureus protein A induces airway epithelial inflammatory responses by activating TNFR1. Nat Med. 2004;10(8):842-848.

7. Corrigan RM, Miajlovic H, Foster TJ. Surface proteins that promote adherence of Staphylococcus aureus to human desquamated nasal epithelial cells. BMC Microbiol. 2009;9:22.
8. Barbu EM, Mackenzie C, Foster TJ, Höök M. SdrC induces staphylococcal biofilm formation through a homophilic interaction. Mol Microbiol. 2014;94(1):172-185

9. Feuillie C, et al. Molecular interactions and inhibition of the staphylococcal biofilm-forming protein SdrC. Proc Natl Acad Sci U S A. 2017;114(14):3738-3743.

10. Gross M, Cramton SE, Götz F, Peschel A. Key role of teichoic acid net charge in Staphylococcus aureus colonization of artificial surfaces. Infect Immun. 2001;69(5):3423-3426.

11. Lam H, Kesselly A, Stegalkina S, Kleanthous H, Yethon JA. Antibodies to PhnD inhibit staphylococcal biofilms. Infect Immun. 2014;82(9):3764-3774.

12. Inoshima I, et al. A Staphylococcus aureus pore-forming toxin subverts the activity of ADAM10 to cause lethal infection in mice. Nat Med. 2011;17(10):1310-1314.

13. Kitur K, et al. Toxin-induced necroptosis is a major mechanism of Staphylococcus aureus lung damage. PLoS Pathog. 2015;11(4):e1004820.

14. Chen BB, et al. E3 ligase subunit Fbxo15 and PINK1 kinase regulate cardiolipin synthase 1 stability and mitochondrial function in pneumonia. Cell Rep. 2014;7(2):476-487.

15. Bjarnsholt T, et al. The in vivo biofilm. Trends Microbiol. 2013;21(9):466-474.

16. Nicholas TE. Pulmonary surfactant: no mere paint on the alveolar wall. Respirology. 1996;1(4):247-257.
17. Goldstein E, Lippert W, Warshauer D. Pulmonary alveolar macrophage. Defender against bacterial infection of the lung. J Clin Invest. 1974;54(3):519-528.

18. Lindert J, Perlman CE, Parthasarathi K, Bhattacharya J. Chloride-dependent secretion of alveolar wall liquid determined by opticalsectioning microscopy. Am J Respir Cell Mol Biol. 2007;36(6):688-696.

19. Westphalen K, Monma E, Islam MN, Bhattacharya J. Acid contact in the rodent pulmonary alveolus causes proinflammatory signaling by membrane pore formation. Am J Physiol Lung Cell Mol Physiol. 2012;303(2):L107-L116.

20. Islam MN, et al. Mitochondrial transfer from bone-marrow-derived stromal cells to pulmonary alveoli protects against acute lung injury. Nat Med. 2012;18(5):759-765.

21. Westphalen K, et al. Sessile alveolar macrophages communicate with alveolar epithelium to modulate immunity. Nature. 2014;506(7489):503-506.

22. Mendes RE, Deshpande LM, Smyth DS, Shop$\sin$ B, Farrell DJ, Jones RN. Characterization of methicillin-resistant Staphylococcus aureus strains recovered from a phase IV clinical trial for linezolid versus vancomycin for treatment of nosocomial pneumonia. J Clin Microbiol. 2012;50(11):3694-3702.

23. Walraven CJ, North MS, Marr-Lyon L, Deming P, Sakoulas G, Mercier RC. Site of infection rather than vancomycin MIC predicts vancomycin treatment failure in methicillin-resistant Staph- 
ylococcus aureus bacteraemia. J Antimicrob Chemother. 2011;66(10):2386-2392.

24. Moore CL, et al. Prediction of failure in vancomycin-treated methicillin-resistant Staphylococcus aureus bloodstream infection: a clinically useful risk stratification tool. Antimicrob Agents Chemother. 2011;55(10):4581-4588.

25. Moore CL, et al. Comparative evaluation of epidemiology and outcomes of methicillinresistant Staphylococcus aureus (MRSA) USA300 infections causing community- and healthcareassociated infections. Int J Antimicrob Agents. 2009;34(2):148-155.

26. Herman-Bausier P, El-Kirat-Chatel S, Foster TJ, Geoghegan JA, Dufrêne YF. Staphylococcus aureus fibronectin-binding protein A mediates cell-cell adhesion through low-affinity homophilic bonds. MBio. 2015;6(3):e00413-e00415.

27. Alicea I, Marvin JS, Miklos AE, Ellington AD, Looger LL, Schreiter ER. Structure of the Escherichia coli phosphonate binding protein $\mathrm{PhnD}$ and rationally optimized phosphonate biosensors. J Mol Biol. 2011;414(3):356-369.

28. Martínez A, Ventouras LA, Wilson ST, Karl DM, Delong EF. Metatranscriptomic and functional metagenomic analysis of methylphosphonate utilization by marine bacteria. Front Microbiol. 2013;4:340.

29. Fey PD, et al. A genetic resource for rapid and comprehensive phenotype screening of nonessential Staphylococcus aureus genes. MBio. 2013;4(1):e00537-e00512.

30. Fowler T, Wann ER, Joh D, Johansson S, Foster TJ, Höök M. Cellular invasion by Staphylococcus aureus involves a fibronectin bridge between the bacterial fibronectin-binding MSCRAMMs and host cell beta1 integrins. Eur J Cell Biol. 2000;79(10):672-679.

31. Walev I, et al. Staphylococcal alpha-toxin kills human keratinocytes by permeabilizing the plasma membrane for monovalent ions. Infect Immun. 1993;61(12):4972-4979.

32. Jonas D, Walev I, Berger T, Liebetrau M, Palmer M, Bhakdi S. Novel path to apoptosis: small transmembrane pores created by staphylococcal alpha-toxin in T lymphocytes evoke internucleosomal DNA degradation. Infect Immun. 1994;62(4):1304-1312.

33. Steinhardt RA, Bi G, Alderton JM. Cell membrane resealing by a vesicular mechanism similar to neurotransmitter release. Science. 1994;263(5145):390-393.

34. Su M, He C, West CA, Mentzer SJ. Cytolytic peptides induce biphasic permeability changes in mammalian cell membranes. JImmunol Methods. 2001;252(1-2):63-71.

35. Wang N, et al. Connexin mimetic peptides inhibit $\mathrm{Cx} 43$ hemichannel opening triggered by voltage and intracellular $\mathrm{Ca}^{2+}$ elevation. Basic Res Cardiol. 2012;107(6):304.

36. Weston SA, Parish CR. New fluorescent dyes for lymphocyte migration studies. Analysis by flow cytometry and fluorescence microscopy. J Immunol Methods. 1990;133(1):87-97.

37. Bubeck Wardenburg J, Schneewind O. Vaccine protection against Staphylococcus aureus pneumonia. J Exp Med. 2008;205(2):287-294.

38. Wilke GA, Bubeck Wardenburg J. Role of a disin- tegrin and metalloprotease 10 in Staphylococcus aureus $\alpha$-hemolysin-mediated cellular injury. Proc Natl Acad Sci U S A. 2010;107(30):13473-13478.

39. Griesbeck O, Baird GS, Campbell RE, Zacharias DA, Tsien RY. Reducing the environmental sensitivity of yellow fluorescent protein. Mechanism and applications. J Biol Chem. 2001;276(31):29188-29194.

40. Palmer AE, Tsien RY. Measuring calcium signaling using genetically targetable fluorescent indicators. Nat Protoc. 2006;1(3):1057-1065.

41. Ichimura $\mathrm{H}$, Parthasarathi K, Quadri S, Issekutz AC, Bhattacharya J. Mechano-oxidative coupling by mitochondria induces proinflammatory responses in lung venular capillaries. JClin Invest. 2003;111(5):691-699.

42. Ichimura H, Parthasarathi K, Lindert J, Bhattacharya J. Lung surfactant secretion by interalveolar $\mathrm{Ca}^{2+}$ signaling. Am J Physiol Lung Cell Mol Physiol. 2006;291(4):L596-L601.

43. Wu S, et al. $\mathrm{Ca}^{2+}$ entry via $\alpha 1 \mathrm{G}$ and TRPV 4 channels differentially regulates surface expression of P-selectin and barrier integrity in pulmonary capillary endothelium. Am J Physiol Lung Cell Mol Physiol. 2009;297(4):L650-L657.

44. Islam MN, Gusarova GA, Monma E, Das SR, Bhattacharya J. F-actin scaffold stabilizes lamellar bodies during surfactant secretion. Am J Physiol Lung Cell Mol Physiol. 2014;306(1):L50-L57.

45. Otsu K, Das S, Houser SD, Quadri SK, Bhattacharya $S$, Bhattacharya J. Concentration-dependent inhibition of angiogenesis by mesenchymal stem cells. Blood. 2009;113(18):4197-4205.

46. Berube BJ, Bubeck Wardenburg J. Staphylococcus aureus $\alpha$-toxin: nearly a century of intrigue. Toxins (Basel). 2013;5(6):1140-1166.

47. Husmann M, et al. Elimination of a bacterial pore-forming toxin by sequential endocytosis and exocytosis. FEBS Lett. 2009;583(2):337-344.

48. Ashino Y, Ying X, Dobbs LG, Bhattacharya J. $[\mathrm{Ca}(2+)](\mathrm{i})$ oscillations regulate type II cell exocytosis in the pulmonary alveolus. Am J Physiol Lung Cell Mol Physiol. 2000;279(1):L5-13.

49. Gehr P, Bachofen M, Weibel ER. The normal human lung: ultrastructure and morphometric estimation of diffusion capacity. Respir Physiol. 1978;32(2):121-140.

50. Weibel ER, Limacher W, Bachofen H. Electron microscopy of rapidly frozen lungs: evaluation on the basis of standard criteria. JAppl Physiol Respir Environ Exerc Physiol. 1982;53(2):516-527.

51. Bastacky J, et al. Alveolar lining layer is thin and continuous: low-temperature scanning electron microscopy of rat lung. J Appl Physiol. 1995;79(5):1615-1628.

52. Echelman DJ, Lee AQ, Fernández JM. Mechanical forces regulate the reactivity of a thioester bond in a bacterial adhesin. J Biol Chem. 2017;292(21):8988-8997.

53. Garmory HS, Titball RW. ATP-binding cassette transporters are targets for the development of antibacterial vaccines and therapies. Infect Immun. 2004;72(12):6757-6763.

54. Leon-Kempis Mdel R, Guccione E, Mulholland F, Williamson MP, Kelly DJ. The Campylobacter jejuni PEB1a adhesin is an aspartate/glutamatebinding protein of an $\mathrm{ABC}$ transporter essential for microaerobic growth on dicarboxylic amino acids.
Mol Microbiol. 2006;60(5):1262-1275.

55. Haaber J, Cohn MT, Frees D, Andersen TJ, Ingmer H. Planktonic aggregates of Staphylococcus aureus protect against common antibiotics. PLoS One. 2012;7(7):e41075.

56. Dastgheyb S, Parvizi J, Shapiro IM, Hickok NJ, Otto M. Effect of biofilms on recalcitrance of staphylococcal joint infection to antibiotic treatment. J Infect Dis. 2015;211(4):641-650.

57. Vardakas KZ, Matthaiou DK, Falagas ME. Incidence, characteristics and outcomes of patients with severe community acquired-MRSA pneumonia. Eur Respir J. 2009;34(5):1148-1158.

58. Khawaja A, Zubairi AB, Durrani FK, Zafar A Etiology and outcome of severe community acquired pneumonia in immunocompetent adults. BMC Infect Dis. 2013;13:94.

59. Ridger VC, Wagner BE, Wallace WA, Hellewell PG. Differential effects of CD18, CD29, and CD49 integrin subunit inhibition on neutrophil migration in pulmonary inflammation. J Immunol. 2001;166(5):3484-3490.

60. Kiefmann R, Islam MN, Lindert J, Parthasarathi $\mathrm{K}$, Bhattacharya J. Paracrine purinergic signaling determines lung endothelial nitric oxide production. Am J Physiol Lung Cell Mol Physiol. 2009;296(6):L901-L910.

61. Wang PM, Ashino Y, Ichimura H, Bhattacharya J. Rapid alveolar liquid removal by a novel convective mechanism. Am J Physiol Lung Cell Mol Physiol. 2001;281(6):L1327-L1334.

62. Selinger SL, Bland RD, Demling RH, Staub NC. Distribution volumes of [131I] albumin, [14C] sucrose, and $36 \mathrm{Cl}$ in sheep lung. J Appl Physiol. 1975;39(5):773-779.

63. Bhattacharya J, Cruz T, Bhattacharya S, Bray BA. Hyaluronan affects extravascular water in lungs of unanesthetized rabbits. JAppl Physiol. 1989;66(6):2595-2599.

64. Safdar Z, Yiming M, Grunig G, Bhattacharya J. Inhibition of acid-induced lung injury by hyperosmolar sucrose in rats. Am J Respir Crit Care Med. 2005;172(8):1002-1007.

65. Crandon JL, Kuti JL, Nicolau DP. Comparative efficacies of human simulated exposures of telavancin and vancomycin against methicillin-resistant Staphylococcus aureus with a range of vancomycin MICs in a murine pneumonia model. Antimicrob Agents Chemother. 2010;54(12):5115-5119.

66. Labrou M, Michail G, Ntokou E, Pittaras TE, Pournaras S, Tsakris A. Activity of oxacillin versus that of vancomycin against oxacillin-susceptible mecA-positive Staphylococcus aureus clinical isolates evaluated by population analyses, time-kill assays, and murine thigh infection model. Antimicrob Agents Chemother. 2012;56(6):3388-3391.

67. Frank JA, Briot R, Lee JW, Ishizaka A, Uchida T, Matthay MA. Physiological and biochemical markers of alveolar epithelial barrier dysfunction in perfused human lungs. Am J Physiol Lung Cell Mol Physiol. 2007;293(1):L52-L59.

68. Traber KE, et al. agr function in clinical Staphylococcus aureus isolates. Microbiology (Reading, Engl). 2008;154(pt 8):2265-2274.

69. Stulik L, et al. $\alpha$-Hemolysin activity of methicillinsusceptible Staphylococcus aureus predicts ventilator-associated pneumonia. Am J Respir Crit Care Med. 2014;190(10):1139-1148. 